MODELING, IDENTIFICATION AND CONTROL, 2001, VOL. 22, NO. 3, 153-187

doi:10.4173/mic.2001.3.3

\title{
Optimal Operation of Petlyuk Distillation: Steady-State Behavior*
}

\author{
IVAR J. HALVORSEN $\dagger$ and SIGURD SKOGESTAD ††
}

Keywords: self optimizing control, distillation

\begin{abstract}
The "Petlyuk" or "dividing-wall" or "fully thermally coupled" distillation column is an interesting alternative to the conventional cascaded binary columns for separation of multi-component mixtures. However, the industrial use has been limited, and difficulties in operation have been reported as one reason. With three product compositions controlled, the system has two degrees of freedom left for on-line optimization. We show that the steady-state optimal solution surface is quite narrow, and depends strongly on disturbances and design parameters. Thus it seems difficult to achieve the potential energy savings compared to conventional approaches without a good control strategy. We discuss candidate variables which may be used as feedback variables in order to keep the column operation close to optimal in a "self-optimizing" control scheme.
\end{abstract}

\section{Introduction}

The thermally integrated "Petlyuk" arrangement has several appealing features. For the separation of a three-component mixture, Triantafyllou and Smith (1992) report typical savings in the order of $30 \%$ in both energy and capital costs compared to traditional arrangements with two columns in series. However, an important question remains: Is this process units difficult to operate and is it possible to achieve in practice the energy savings?

The Petlyuk column, shown in Figure 1, has at steady state five degrees of freedom, which may be selected as the following manipulative inputs: Boilup $(V)$, reflux $(L)$, mid product side-stream flow $(S)$, liquid split $\left(R_{l}=L_{1} / L\right)$ and vapor split $\left(R_{v}=V_{2} / V\right)$. There may be up to four product specifications: Top purity $\left(x_{D a}\right)$, bottoms purity $\left(x_{B c}\right)$, side-stream purity $\left(x_{S b}\right)$ and the ratio of the light and heavy impurity components in the side-stream product $\left(x_{S a} / x_{S c}\right)$. However, Wolff et al. $(1994,1996)$ have reported discontinuities in the range of feasible operation if all these product compositions are specified. This is related to the fact that column sections 4 and 5 (see Figure 1) are tightly coupled and we cannot independently adjust the amount of light and heavy component in the intermediate side-stream product. This may be a disadvantage compared to a conventional arrangement with two columns. On the other hand, if the number of controlled outputs is reduced from four to three, by not considering the ratio of light/heavy impurity-components in the side-stream, the feasibility problem disappears. Thus in this paper we will focus on this simpler task of three-point control, where the purities of the main component in each product are specified $\left(x_{D a}, x_{B c}, x_{S b}\right)$. The remaining extra two degrees of freedom

* Reprinted from Journal of Process Control, Vol. 9, No. 5, 1999, pages 407-424, Copyright 1999, with permissions from Elsevier Science

†SINTEF Electronics and Cybernetics, 7465 Trondheim, Norway

$\dagger+$ Norwegian University of Science and Technology, Department of Chemical Engineering, 7491 Trondheim, Norway 


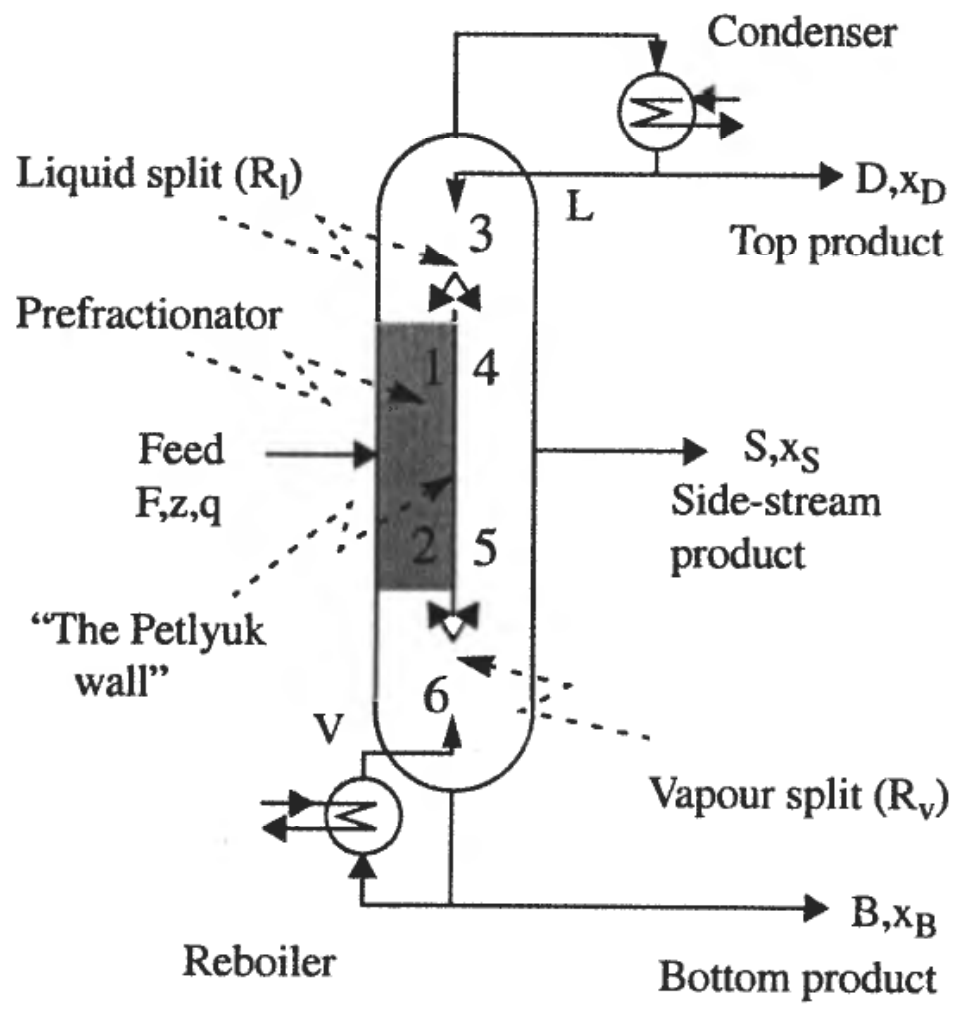

Figure 1. The Petlyuk Distillation Column implemented in a single column shell.

can then be used for other purposes, and in particular for minimizing the operating cost, which in our case is the energy consumption $(V)$.

The practical problem of keeping operation at optimum is illustrated in Figure 2 which may represent the energy consumption $V$ (Criterion) as a function of the liquid split $R_{l}$ (Free control variable). We are nominally operating at the optimum but then

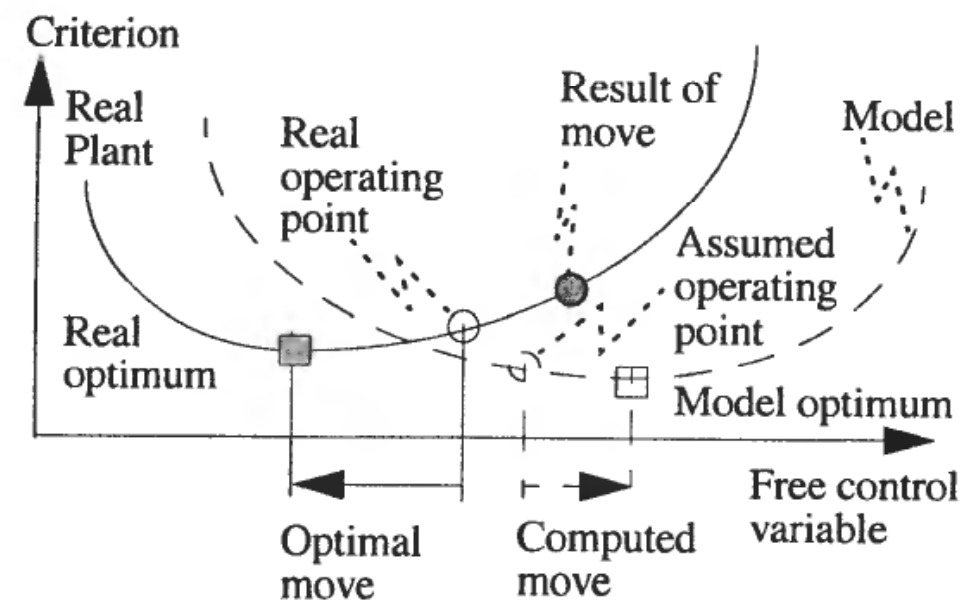

Figure 2. Optimization problems with unknown disturbances and model uncertainties. 
the optimal operating point has moved due to some unknown disturbance, and we want to compute the optimal move in our available manipulative variable in order to follow the real optimum. With model uncertainty and unknown disturbances it may be difficult to tell in which direction the free variable should be moved in order to bring the process closer to the real optimum.

Three main approaches to deal with this problem are: model based methods, experimenting methods (e.g. EVOP) and feedback methods. In this paper we will focus on the feedback method. This is the simplest method, requiring the least modeling effort for implementation, and is therefore the preferred choice if it gives acceptable performance. In our case the objective is to use the two extra manipulated inputs (e.g. $R_{l}$ and $R_{v}$ ) to minimize the energy consumption per unit feed $(V / F)$. The key step for the feedback method is to translate this optimization problem into a setpoint problem. The issue is then to find a set of variables which, when kept constant at their setpoints, indirectly ensures optimal operation. Figure 3 illustrates this idea.

Since the criterion function $(V)$ in our case is also a possible free variable, one seemingly viable solution for the Petlyuk column would be to simply implement the optimal minimum heat input in an open loop fashion, i.e. to perform an optimization to compute the minimum of $V$ with respect to the degrees of freedom $\left(u_{D O F}\right)$.

$$
V_{0}=\min _{u_{D O F}} V
$$

and then simply set $V=V_{0}$. However, there are at least three serious problems:

1. Operation is infeasible for $V<V_{0}$, so we would need to use $V>V_{0}$.

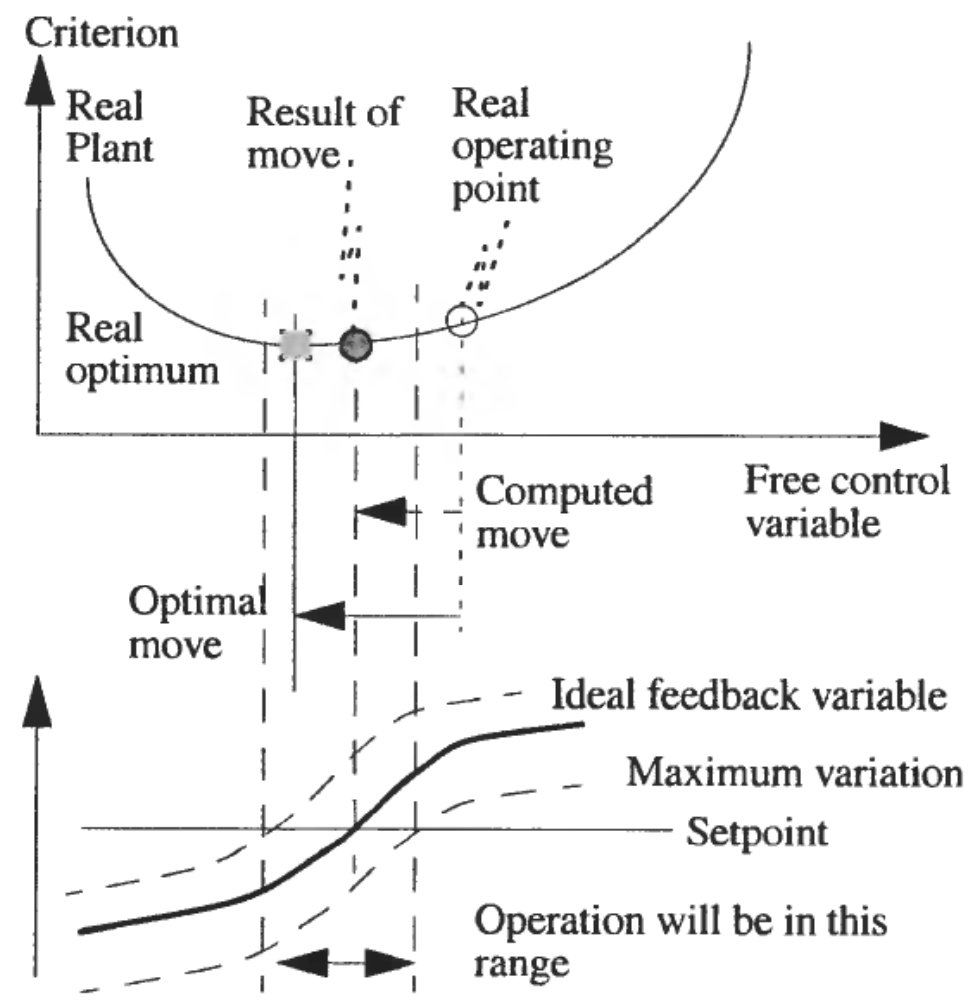

Figure 3. Optimization by controlling a suitable feedback variable to a setpoint. 
2. The optimal value of $V_{0}$ changes with operation, and it would require a good model and measurements of the disturbances to recompute it.

3. Measurement or estimation of the actual $V$ is generally difficult and inaccurate, which makes it even more difficult to keep $V$ close to $V_{0}$.

Thus, this open-loop policy is clearly not viable. As good candidate variables for feedback control we want variables which avoid the three problems above and satisfies the following requirements:

1. The optimal candidate feedback value should not be at an unconstrained extremum (like $V=V_{0}$ ).

2. The optimal value of the variable should be insensitive to disturbances.

3. The accuracy of the measurement of the variable should be good and the variable should be easy to the control, using the available extra degrees of freedom.

Often we may find variables which have an extremum when the criterion functions is at its minimum. Although these cannot be used for feedback, they may be used in experimental methods, or as indicators to process operators.

A variable related to the gradient of the criterion function fulfills requirements 1 and 2 .

In general it is not always possible to find a feedback variable with the required property of turning the optimization problem into a setpoint problem. However, for processes with a large number of states, and a large number of ways to combine measurements, good candidates may exist, but they may not be easy to find. Skogestad and Postlethwaite (1996) present a method for selecting the best candidate feedback variables from a set of available alternatives. (See their remark on page 405.) We will not consider this procedure here, but rather aim at obtaining insight into the column behavior that may be used for selecting candidate feedback variables.

Some interesting questions for the Petlyuk column are: which variables should be used as the degrees of freedom in order to achieve the best practical result? (The choice $\left(R_{l}, R_{v}\right)$ mentioned above is not necessarily the best.) Can we leave both degrees of freedom constant? Or can we leave one constant and use the other one for our optimization task? Or do we need to use both degrees of freedom for on-line optimization? How large changes in disturbances can we accept?

\section{The Petlyuk column model}

We use a stage-by-stage model with the following simplifying assumptions: constant pressure, equilibrium stages with constant relative volatilities, constant molar flows, no heat transfer through the dividing wall. This model is very simple, but it contains the most important properties of a column. The model and column data are given in Table 1. Since we focus on the steady-state properties we do not need to include data for tray and condenser holdups.

To model the column in Figure 1 we use 6 sections of stages (the numbers inside the column are section numbers). In our case study a three-component (ternary) feed, consisting of components $a, b$ and $c$ is separated into almost pure $a(97 \%)$ in the top product $\mathrm{D}$, almost pure $b(97 \%)$ in the side stream $\mathrm{S}$, and almost pure $c(97 \%)$ in the bottom product $\mathrm{B}$.

The input, output and disturbance vectors are defined next. There are five degrees of freedom which we select as the following manipulated inputs:

$$
u=\left[L, V, S, R_{l}, R_{v}\right]
$$


Table 1. Optimal steady-state solution

\begin{tabular}{lc}
\hline Parameter//Variable & Base case \\
\hline Relative volatility $\left[\alpha_{A}, \alpha_{B}, \alpha_{c}\right]$ & {$[4,2,1]$} \\
Feed composition $\left[z_{a}, z_{b}, z_{b}\right]$ & {$[1 / 31 / 31 / 3]$} \\
Feed liquid fraction $q$ & $0 \cdot 477$ \\
$y_{s}=\left[x_{D a}, x_{S b}, x_{B c}\right]$ & {$[0 \cdot 97,0 \cdot 97,0 \cdot 97]$} \\
& \\
$u_{1 . o p t}=\left[R_{l}, R_{v}\right]$ & {$[0 \cdot 450,0 \cdot 491]$} \\
$V_{o p t}$ & $1 \cdot 498$ \\
$x_{\text {Sa }} / x_{S c}$ & $0 \cdot 937$ \\
\hline
\end{tabular}

Three outputs (compositions) are controlled:

$$
y=\left[d_{D a}, x_{B c}, x_{S b}\right]
$$

The disturbances associated with the feed are:

$$
d=\left[F, z_{a}, z_{b}, q\right]
$$

In addition to the outputs in $y$, we will propose later some other measurements to be used for optimization purposes. We will also present results from a model where we assume infinite number of stages and sharp product splits, but with the same feed.

\section{Optimization criterion}

We assume that it is optimal to keep the product purities at their specifications (i.e. the setpoints are $97 \%$ purity). This is reasonable in most cases unless the product values are very different or energy is very cheap. The column has 5 degrees of freedom at steady-state so with 3 setpoints specified we have 2 degrees of freedom left for optimization. We choose as a base case the two remaining degrees of freedom to be $R_{l}$ and $R_{v}$. (Note that other choices could have been made.)

With the three product purities given, the only operation variables that affect the operating costs are the reboiler and condenser duty. Both are proportional to the boilup rate $V$, and as the optimization criterion we therefore choose to minimize the scalar "cost" $J=V I F$. (We normalize the throughput $(F=1)$ and minimizing $V I F$ is then equivalent to minimizing $V$.)

With our assumptions the steady state optimization problem can be written on the following general form:

$$
\min _{u_{1}} J=\min _{u_{1}} V\left(u_{1}, y_{s}, d\right)=V_{\text {opt }}\left(y_{s}, d\right)
$$

where $u_{1}=\left[R_{l}, R_{v}\right]$ denote the degrees of freedom. The other three manipulated inputs $u_{2}=[L, V, S]$ are not degrees of freedom any more since their values are determined indirectly by the product purity setpoints $\left(y_{s}\right)$ and $u_{1}$. The solution to equation (2) yields the optimal values of the degrees of freedom as a function of the external disturbances $(d)$ and the product specifications $\left(y_{s}\right)$,

$$
u_{1, o p t}=U\left(y_{s}, d\right)
$$

In many optimization problems, the optimal solution is at some "active" constraint(s), and the optimizing control task can be reduced to controlling the active constrained variables. However, for our application the optimal solution is usually 
not at a constraint. Thus, the optimal solution to the problem in equation (2) is a point where the gradient $\nabla V_{u_{1}}=0$ which usually is much more difficult to find and implement. The reason is that we do not really know the disturbances $d$ accurately, and unless we have a very good model we do not even know the function to be minimized in equation (2).

We will leave this problem for a while, and assume that we know the model and the disturbances, and we will investigate the shape of the cost function $(J=V)$, that is, how it depends on changes in product purity specifications and disturbances.

\subsection{Criterion with state space model}

With a stage-by-stage model, we can formulate the criterion with the model equation included as equality constraints:

$$
\min _{[x, u]} J=V
$$

subject to the constraints

$$
\begin{gathered}
f(x, u, d)=0 \\
h\left(x, u, d, y_{s}\right) \leqslant 0
\end{gathered}
$$

Here $f$ is the column model and $h$ is a set of equality or inequality constraints. The states $(x)$ consist of two component compositions on each equilibrium stage. For our column, the total number of states is 100 (there are 48 stages plus reboiler and condenser). Typically, $h$ will contain product specifications (e.g. $\left.x_{D a}>0.97\right)$ and other operational constraints like an allowed range for the inputs $u$ (e.g. $u_{\min } \leqslant u \leqslant$ $u_{\max }$ ) and internal flow constraints, e.g. to avoid flooding. (The latter constraints are not considered here, but such problems have to be dealt with in industrial columns.)

It is important to note that the problems and solutions for equations (2) and (4) are identical. The difference is that with equation (4) we get the solution expressed by the full state and input vector $[x, u]$ and we can easily use our model equations directly.

\section{Results from the model case study}

\subsection{Optimal steady state profiles}

We here consider the optimal steady state solution with three compositions specified and with the two remaining degrees of freedom chosen such that the vapor boilup $V$ (energy consumption) is minimized. The results for our base case are shown in Table 1.

Figure 4a shows the resulting optimal composition profiles along the column for the base case in Table 1 and optimal profiles for various feed disturbances is shown in Figure $4 \mathrm{~b}$. We observe that the stage with maximum $b$-composition is the side-stream stage, which intuitively seems reasonable. We also observe that the prefractionator (dashed lines) separates $a$ from $c$ almost completely. Thus we can regard sections $1+2$ as a column of separation of $a$ from $c$, sections $3+4$ as a binary column for separation of $a$ and $b$, and sections $5+6$ as a binary column for separation of $b$ and $c$. The "tricky" part is that the amount of $b$ in the "feeds" to "columns" $3+4$ and $5+6$ depends on the control inputs $u_{1}=\left[R_{l}, R_{v}\right]$, and that we have the same vapor flow from the lower part of the main column through to the upper part (from section 5 to 4).

Normally, composition measurements along the column are not available, but 


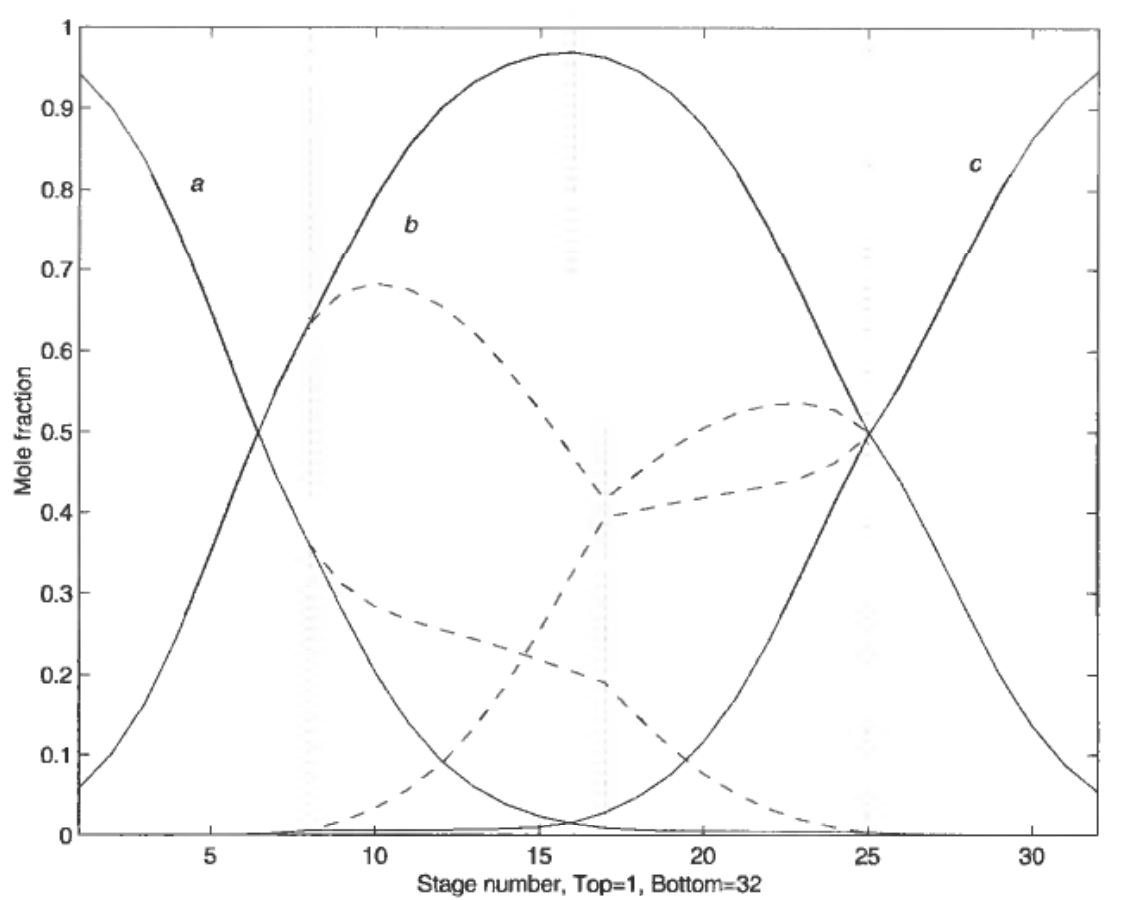

Figure 4a. Optimal composition profiles for components $a, b$ and $c$ in pre-fractionator (dashed) and main column (solid) for the base case in Table 1.

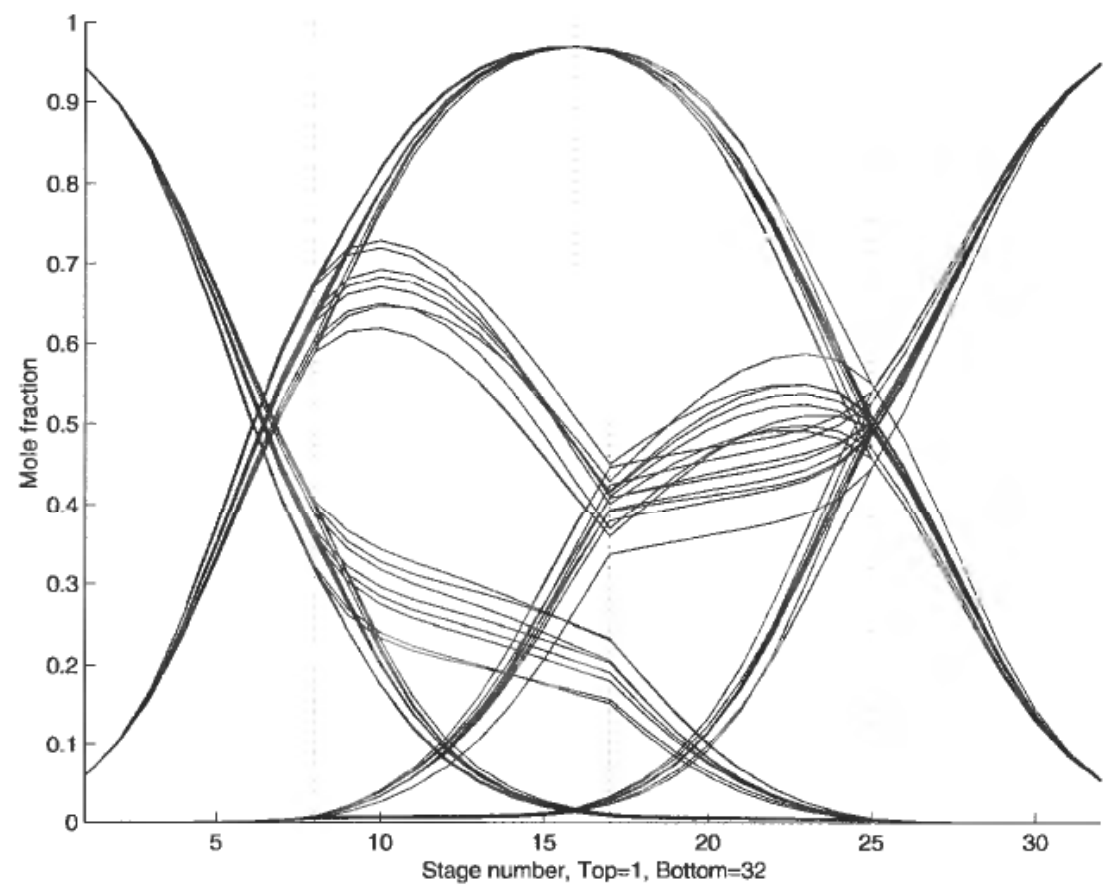

Figure 4b. Optimal composition profiles for various disturbances in the feed composition $( \pm 0.05)$ and the liquid fraction $( \pm 0-1)$. 


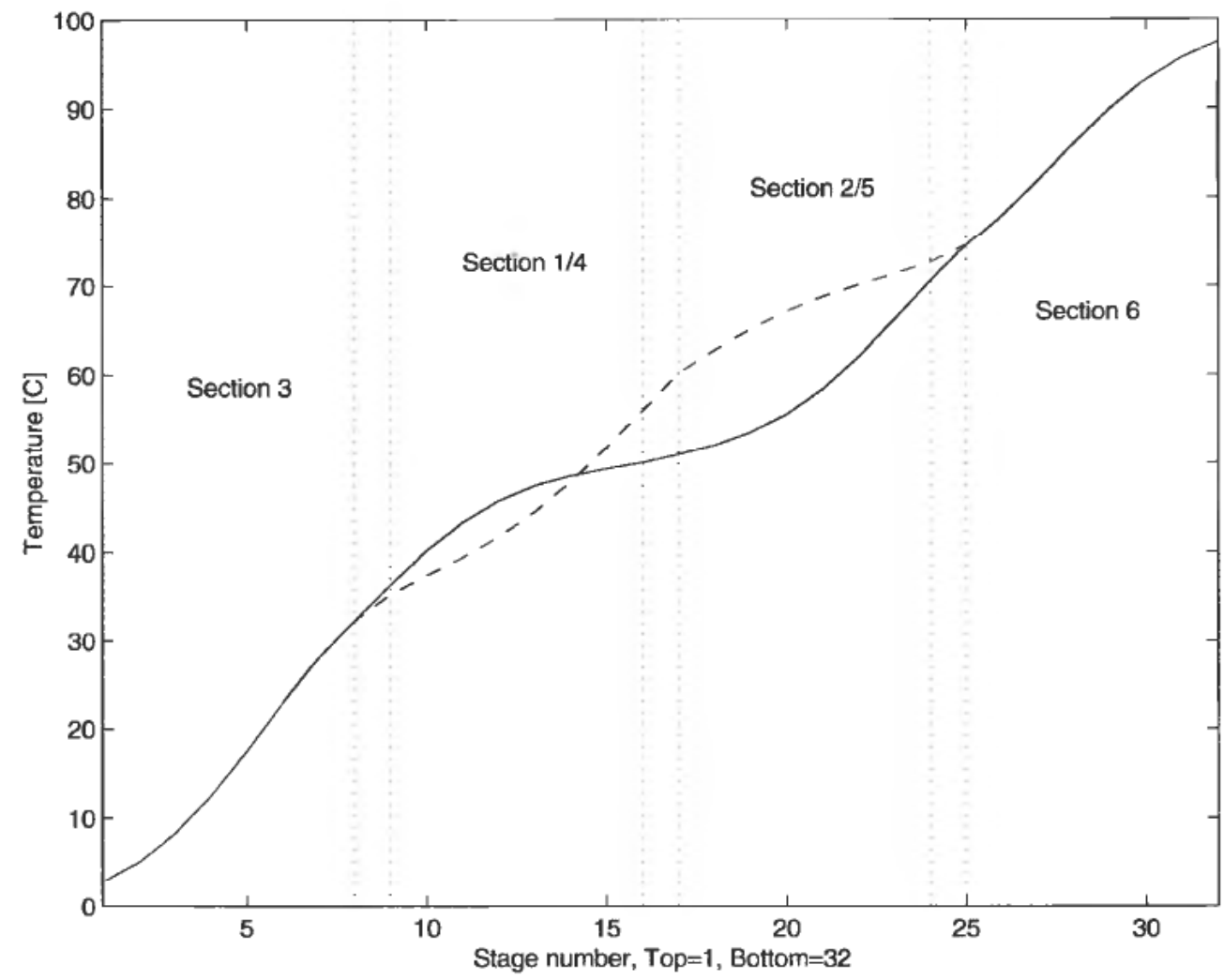

Figure 5. Optimal temperature profile in pre-fractionator (dashed) and main column (solid) for the base case in Table 1 .

temperatures, which are closely related to compositions, may be used to obtain important information. In Figure 5 the temperature profile is shown for a case where the three pure-component boiling points are set to 0,50 and 100 "degrees" for light, medium and heavy component, respectively. At the product locations, the temperature profile is close to the pure product boiling point, and the temperature profile will normally have large gradients where the composition profile has large gradients.

\subsection{The solution surface}

In the following the three product compositions are specified ( $97 \%$ purity). We first study the dependency of the solution surface to variations in $R_{l}$ and $R_{v}$.

$$
V=V\left(R_{l}, R_{v}\right)
$$

This is shown in Figure 6 (surface) and Figure 7 (contour plot) for the base case (which has a partly vaporized feed $q=0 \cdot 48$ ). The surface actually looks like the hull of a ship, and there is a quite flat region ("bottom of the valley") between points $P$ and R. The minimum vapor flow at the "bottom" is $V_{\text {opt }}=1 \cdot 498$, but observe that the vapor flow increase rapidly if we do not keep $\left[R_{l}, R_{v}\right]$ at their optimal values $[0 \cdot 450,0 \cdot 491]$. In the "worst" direction, which is normal to the line PR, the boilup increase by $30 \%$ for a change in $R_{l}$ or $R_{v}$ of just $5 \%$. Whereas, in the "best" direction, along the line PR, we can make a 10 times larger change in $R_{l}$ or $R_{v}(50 \%)$ before 


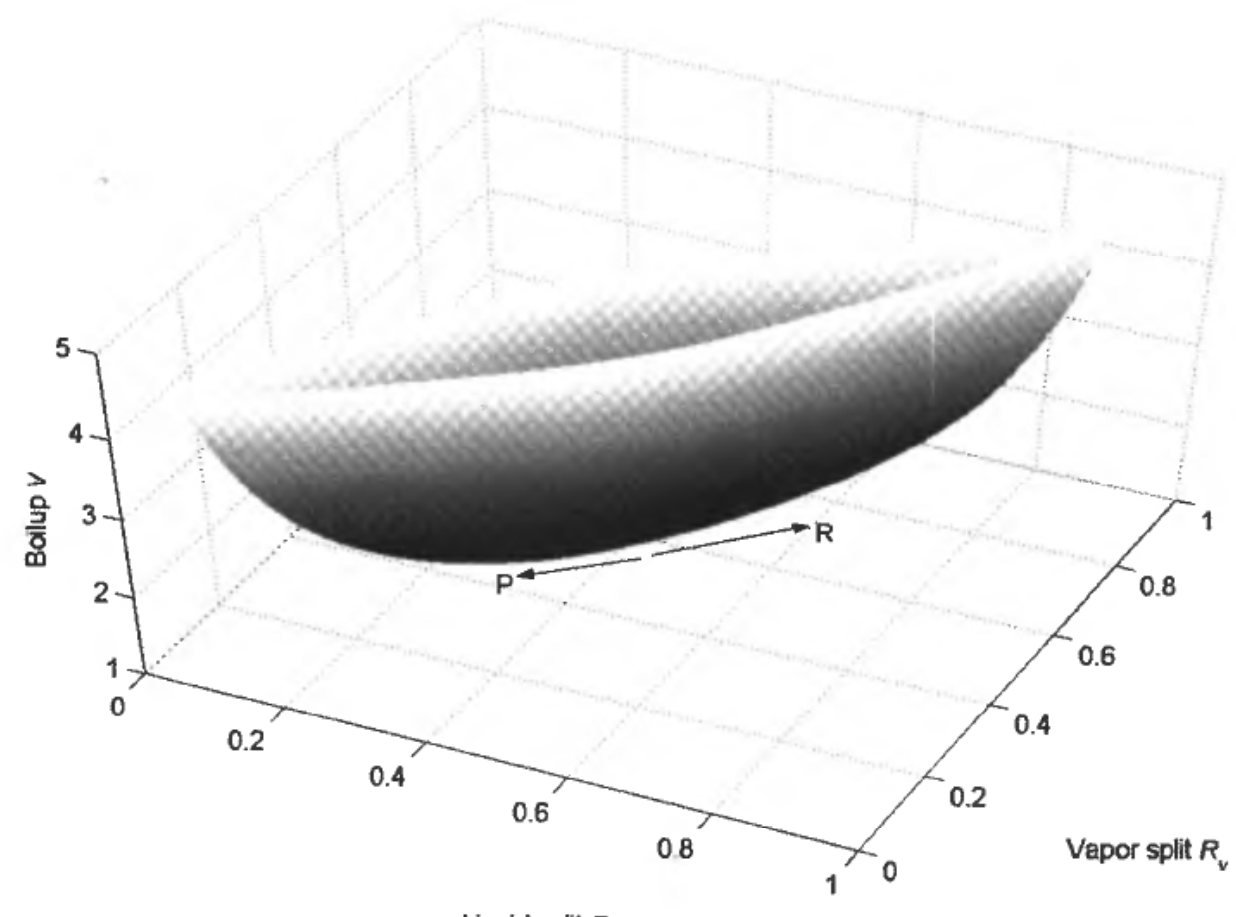

Liquid split $R_{\text {, }}$

Figure 6. Optimal solution surface. $V\left(R_{l}, R_{v}\right)$ (base case).

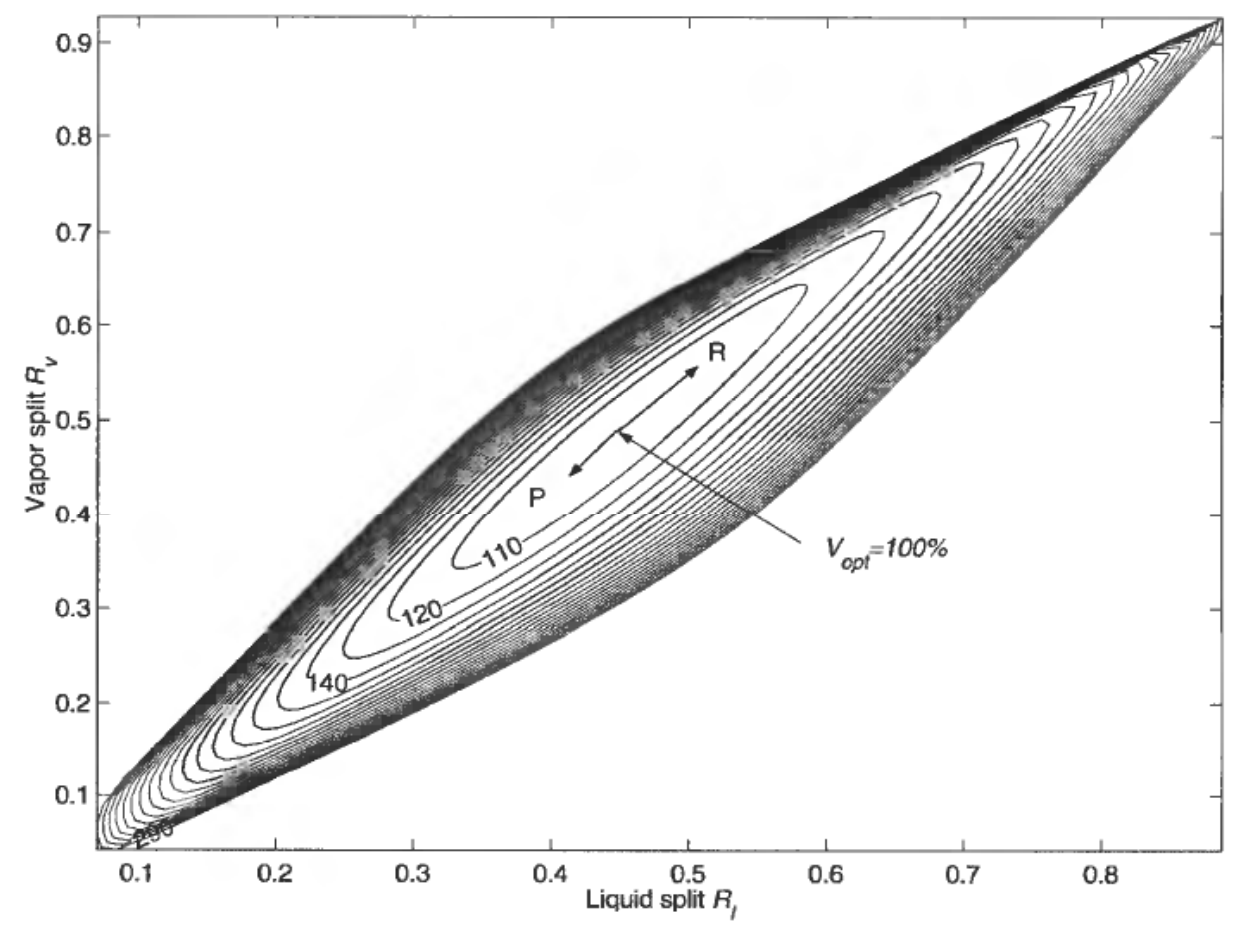

Figure 7. Contour plot of $V$ corresponding to Figure 6. 


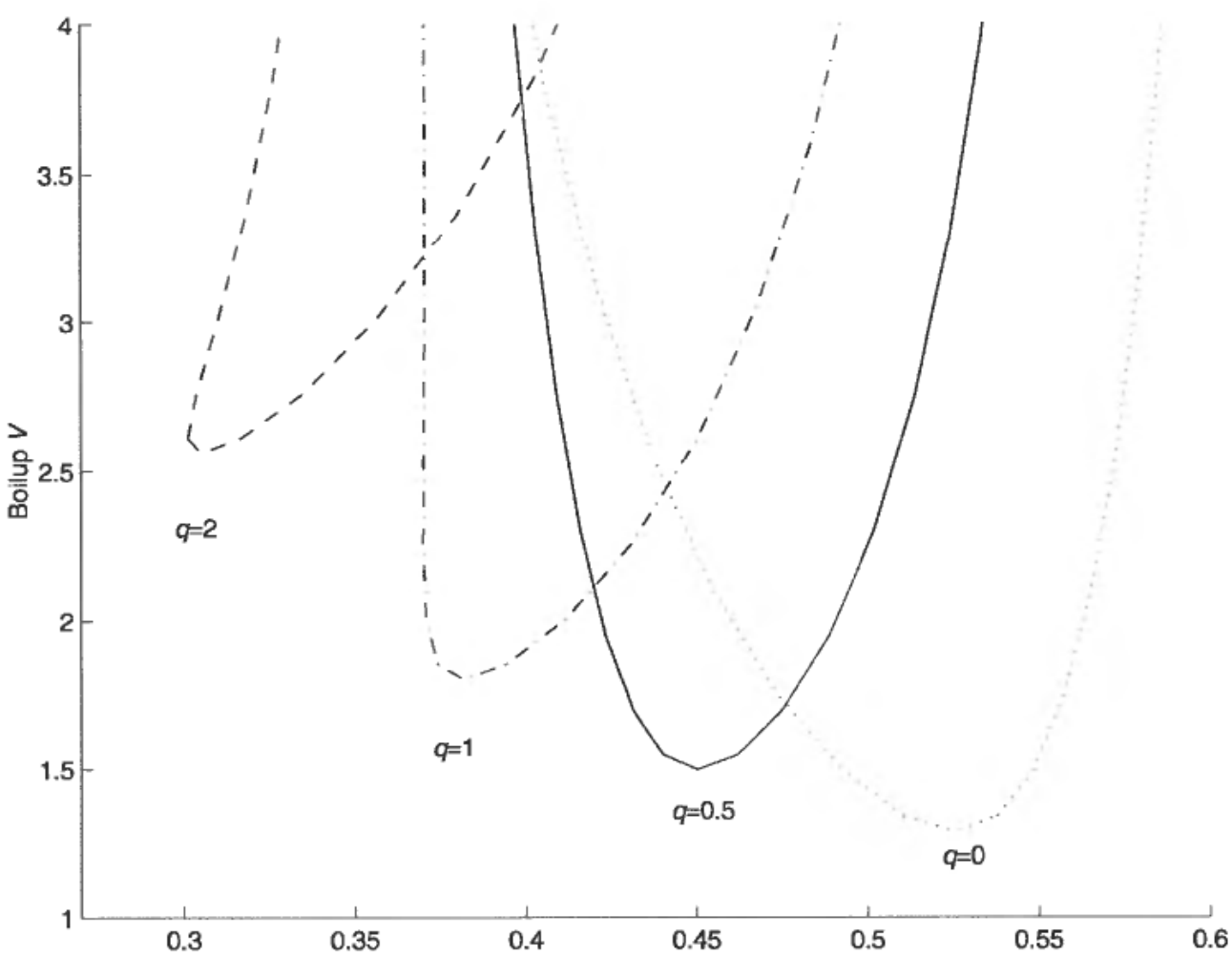

Figure 8. The solution surface for $V$ is very steep and depends strongly on $R_{l}$ in the "bad" direction normal to PR. The whole surface is also strongly dependent on the feed liquid fraction $(q)$.

the boilup increases by $30 \%$. This is further illustrated in Figures 8 and 9 which give cross-sections of the surface in the bad and good directions respectively. We note that for the case with $q=1$, a reduction of $R_{l}$ by just $2 \%$ in the bad direction results in infinite boilup.

The conclusion of this is that at least one of the two degrees-of-freedom $\left(R_{l}\right.$ or $R_{v}$ ) have to be adjusted during operation in order to be able to keep the energy consumption close to its minimum (i.e. operate along the line PR). But it seems possible that one degree of freedom, for instance $R_{v}$, can be left uncontrolled (constant), provided that the other degree of freedom, $R_{l}$, is adjusted to keep the operating point along the "bottom of the valley" (along PR).

\subsection{Effect of disturbances}

If disturbances move the optimum in the "bad" direction normal to PR, then this results in large increases in $V$ unless we adjust $R_{l}$ and/or $R_{v}$ in order to remain in the "bottom of the valley". We find in our case that changes in feed liquid fraction $(q)$, middle feed component $\left(z_{b}\right)$ and sidestream product composition $\left(x_{S, b}\right)$, will move the optimal operating point in the "bad" direction. The other feed composition changes and setpoint changes will move the operation in the "good" direction along the "bottom of the valley" and thus require less attention. The fact that changes in the feed liquid fraction $(q)$ moves optimum in the bad direction normal to PR is illustrated in Figure 8. 


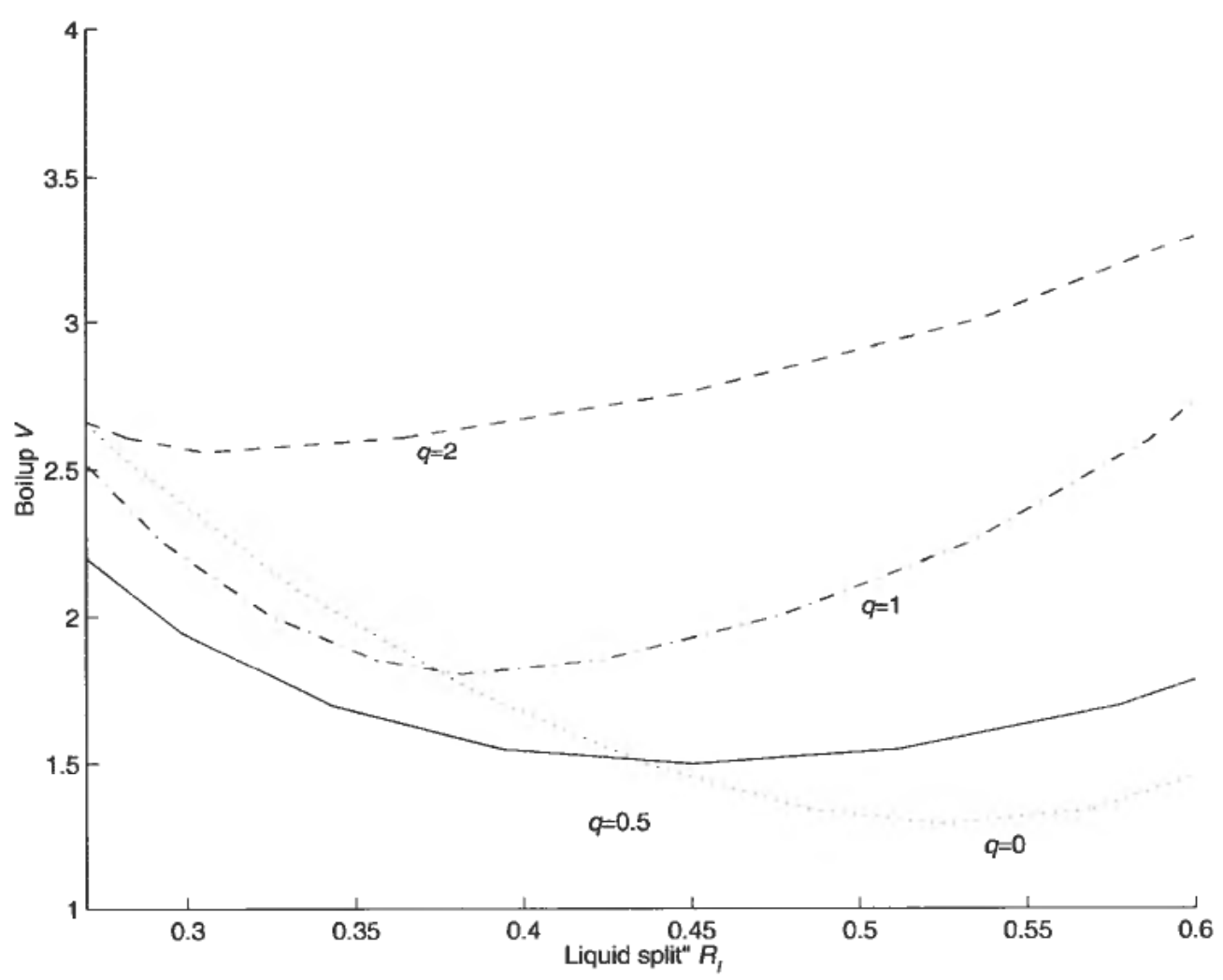

Figure 9. $V$ depends only weakly on $R_{t}$ when $R_{v}$ is adjusted so we stay in the "good" PR-direction. Note that the axis scaling are the same as in Figure 8.

In addition, we see from Figure 8 that changes in $q$ have a dramatic effect on the shape of the solution surface. When the feed is saturated liquid $(q=1)$, the optimal surface becomes almost vertical very close to the optimum. The practical implication of this is that with $R_{l}$ and $R_{v}$ fixed close to their optimal values, the system may become unstable, since we easily may enter a region when there is no feasible solution (no amount of energy can fulfill the composition requirements). For a subcooled liquid $(q>1)$, the solution surface "bends over", and we may have multiple solutions of $V$ for the same product compositions. In open loop, all these operation conditions are reachable and stable. But with composition control active, and tuned for the lower branch, operation on the upper branch is unstable.

Feed flow changes are normally a major disturbance, but do not affect the steady state operation if we keep product compositions $\left(y_{s}\right)$ and split ratios $\left(R_{l}, R_{v}\right)$ constant (since these are all intensive variables). However, feed flow changes will affect the composition control and optimization during a transient.

\subsection{Transport of components}

Interesting insights into the behavior of the column are obtained by considering how each component moves through the column sections towards the products. Define the net upwards flow $w_{j}$ of component $j$ through stage $i$ as:

$$
w_{j}=V_{i} y_{i, j}-L_{i+1} x_{i+1, j}
$$



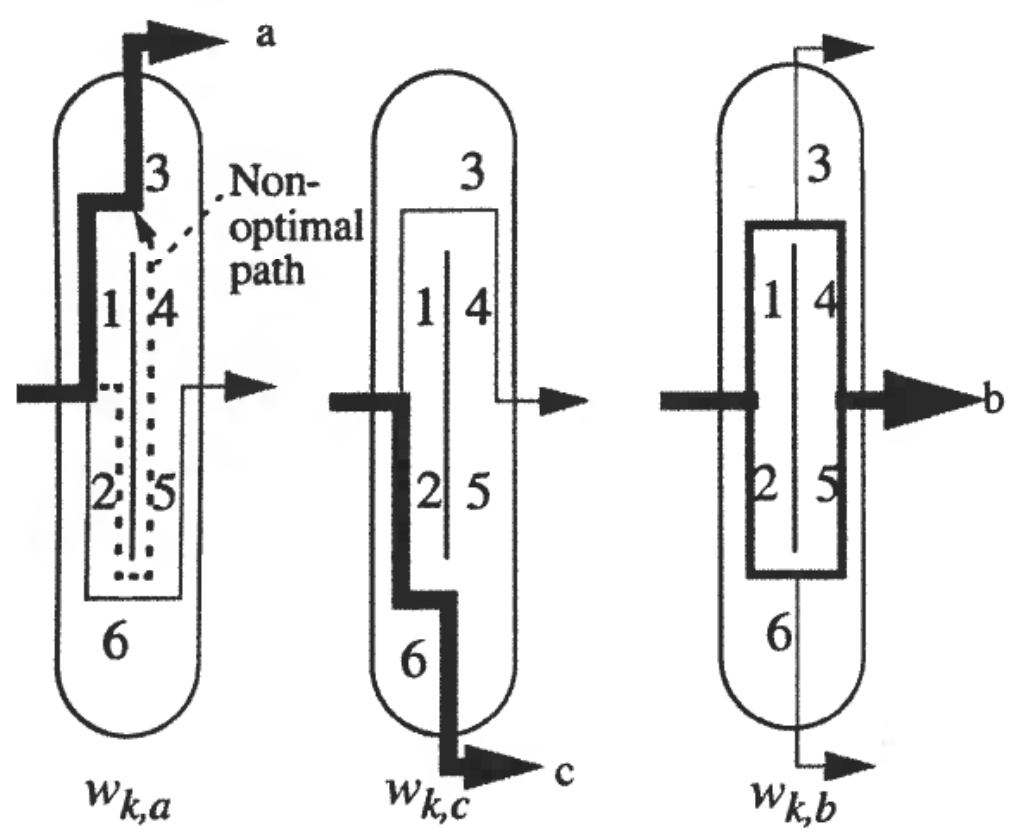

Figure 10. Components taking the "shortest" way.

At steady state $w_{j}$ is constant through each section $k$. The ratio of $w_{k, j}$ to the amount in the feed is the recovery:

$$
r_{k, j}=\frac{w_{k, j}}{F z_{j}}
$$

At optimal operation we find that the component flows $\left(w_{k, j}\right)$ are as indicated in Figure 10. For example, if we look at the light $a$-component, then most of the flow takes the "shortest" way out to the top product. Some light product "slips" down the prefractionator and this mostly ends up in the side stream. Interestingly, for the optimal solution there is no net flow of light component downwards in the section above the side stream, that, is $w_{4, a}$ is close to zero. For the heavy component $(c)$ the behavior is similar, but reversed. The intermediate $b$-component distribute quite evenly along the two paths.

In the following we will in particular consider the effect of changing the recovery $(\beta)$ of component $b$ at the top of the prefractionator:

$$
\beta=r_{1, b}=\frac{w_{1, b}}{F z_{b}}
$$

\section{Analysis from model with infinite number of stages}

The limiting case with an infinite number of stages in each column section provides a lower bound $\left(V_{\min }\right)$ on the energy usage. Although this value cannot be achieved in practice, one can usually come within $10-20 \%$ of the lower bound, so it provides very useful information also for practical distillation. The advantage of using infinite number of stages is that one does not need to consider the issue of selecting the number of stages. Furthermore, excellent theoretical results for the 
Petlyuk column have been presented by Fidkowski and Krolikowski (1986). Through careful treatment of the Underwood equations, they have shown that the minimum energy solution for the Petlyuk column is obtained by operating the prefractionator along its minimum energy characteristic in the range between the preferred split, Stichlmair (1988), and up to a point where the upper and lower part of the main column are balanced.

Christiansen and Skogestad (1977) derived similar results for the closely related case with a separate prefractionator (with its own reboiler and condenser), and they suggested a control structure based on controlling either the impurity of heavy key at the top of the prefractionator, or the impurity of light key at the prefractionator bottom. (The particular choice depends on whether the upper or lower parts of the main column determine minimum reflux.)

We will now use the case with infinite stages to study more carefully how various disturbances and other parameters affect the task of keeping the operation point close to the optimum.

\subsection{Minimum energy consumption for a Petlyuk column}

We first recapitulate the most important results from Fidkowski and Krolikowski (1986). Their results are derived for a saturated liquid $(q=1)$ ternary feed, constant relative volatilities, constant molar flows, infinite number of stages and sharp splits. In Halvorsen (2001) we have extended Fidkowski's result to handle any liquid fraction $(q)$. Fidkowski and Krolikowski use the recovery of the middle component in the net flow out of the top of the prefractionator $(\beta)$ and the "reflux" into the prefractrionator $\left(L_{1}\right)$ as the two degrees of freedom. We will later map $\beta$ and $L_{1}$ to our choice of degrees of freedom, $R_{l}$ and $R_{v}$. Note that minimizing the main column boilup $(V)$ is equivalent to minimizing the main column reflux $(L)$.

At minimum reflux $\left(L_{\min }\right)$ for the Petlyuk column, minimum reflux constraints have to be satisfied for both columns in Figure 1: in the prefractionator (section $1+2$ ), and in either the upper (section $3+4$ ) or lower (sections $5+6$ ) parts of the main column.

First consider the prefractionator which separates the ternary $a b c$-mixture into $a b$ and $b c$. For a sharp split between $a$ and $c$, the minimum reflux $\left(L_{1}\right)$ as a function of the recovery $\beta$ has a distinct minimum at the preferred split $\left(\beta=\beta_{P}\right)$, as shown in Figure 11 for our base case feed.

The main column can be regarded as two binary columns, but their reflux flows are not independent. For large values of $\beta$, most of the $b$-component will have to be separated in the upper part of the main column while the lower part gets an almost pure $c$-feed. Thus the reflux requirement for the upper part of the main column will determine the overall main column reflux and the lower part will be over-refluxed. For low values of $\beta$ we have the opposite case, and for an intermediate value, $\beta=\beta_{R}$, reflux requirements are the same for both parts; at this point the main column is balanced.

\subsection{Solution surface for infinite number of stages}

Fidkowski and Krolikowski (1986) found that the minimum overall reflux $\left(L_{\min }\right)$ is not obtained at a single value of the recovery $\beta$, but rather there is a flat region where $L=L_{\min }$ for a range of recoveries between the preferred split for the 


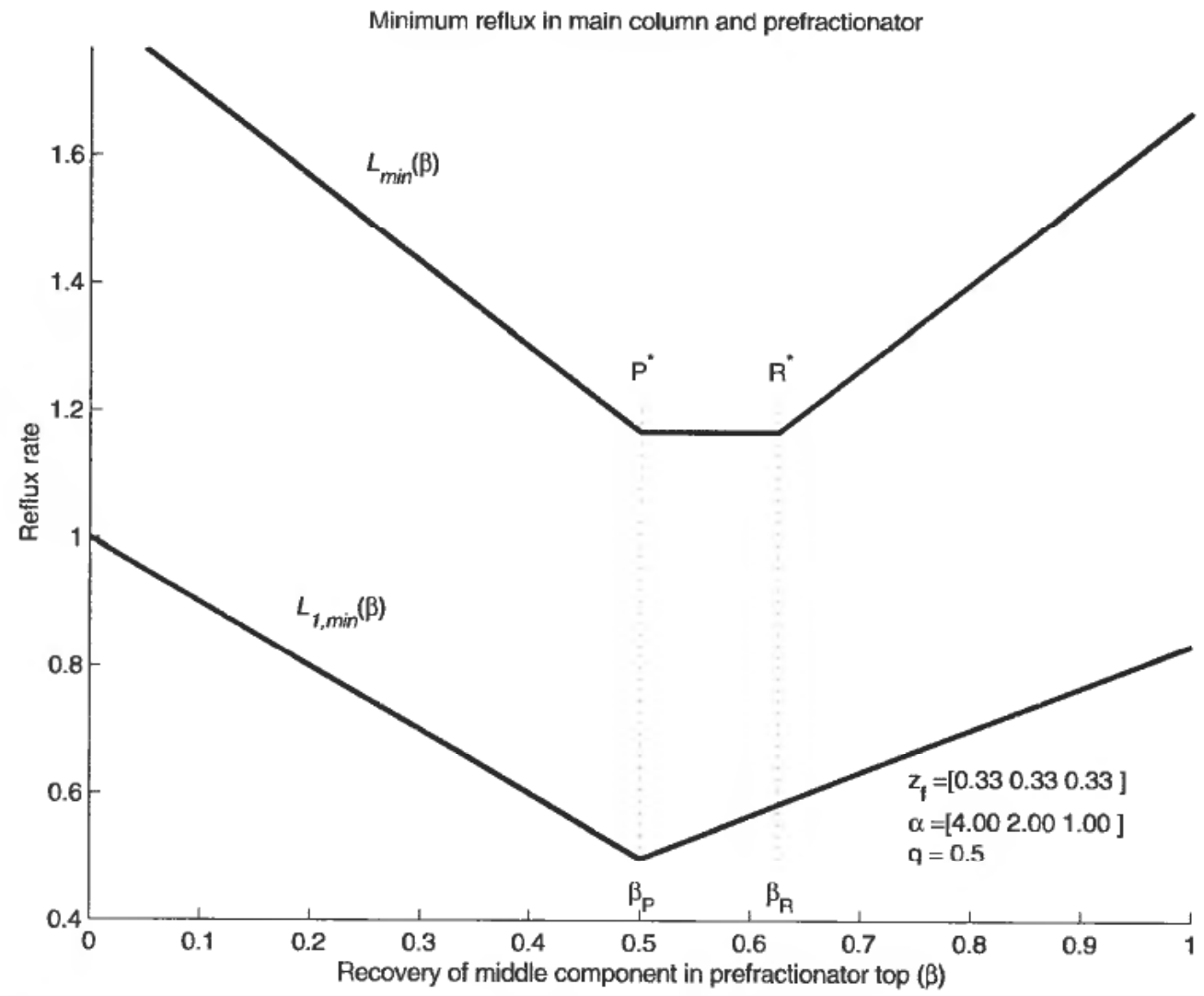

Figure 11. The prefractionator reflux $\left(L_{1}\right)$ has a sharp minimum at the preferred split $\left(\mathrm{P}^{*}\right)$. The overall column reflux $(L)$ is minimal in a flat region $\left(\mathrm{P}^{*}-\mathrm{R}^{*}\right)$ for recoveries between the preferred split $\left(\beta_{P}=0 \cdot 5\right)$ and a balanced main column $\left(\beta_{R}=0 \cdot 625\right)$.

prefractionator $\left(\beta_{P}\right)$, and the value $\left(\beta_{R}\right)$ which makes the main column balanced. This is illustrated in Figure 11.

The flat region may be wide or narrow, depending on the relative values of $\beta_{P}$ and $\beta_{R}$ and we may have cases with either $\beta_{P}>\beta_{R}$ or $\beta_{P}<\beta_{R}$ (like in our example). Only for the special case $\beta_{P} \approx \beta_{R}$ do we have a sharp minimum. Note that the value of $\beta_{P}$ corresponding to the preferred split is always optimal, but depending on the value of $\beta_{R}$, it will be in the left or right end of the flat region.

The corresponding solution surface $V\left(R_{l}, R_{v}\right)$ computed by the infinite stage model and sharp product splits is shown in Figure 12 (surface) and Figure 13 (contour) and is seen to be very similar to the surface for the case study shown previously in Figures 6 and 7.

As already noted, there is a flat region with $V=V_{\min }$ along a straight line from $\mathrm{P}^{*}$ to $\mathrm{R}^{*}$ in the $\left(R_{l}, R_{v}\right)$-plane. The fact that the optimum is flat between $\mathrm{P}^{*}$ and $\mathrm{R}^{*}$ is an important result, and this fully confirms the results based on numerical computations on the column with a finite number of stages.

In appendix A.2 we summarize the results in Halvorsen and Skogestad (1999) and present analytical results for generating the rest of the solution surface. We find that for a given value of the main column boilup $\left(V=\right.$ const, $\left.V>V_{\min }\right)$, the contours in the $\left(R_{l}, R_{v}\right)$-plane are straight lines between four characteristic corner lines 


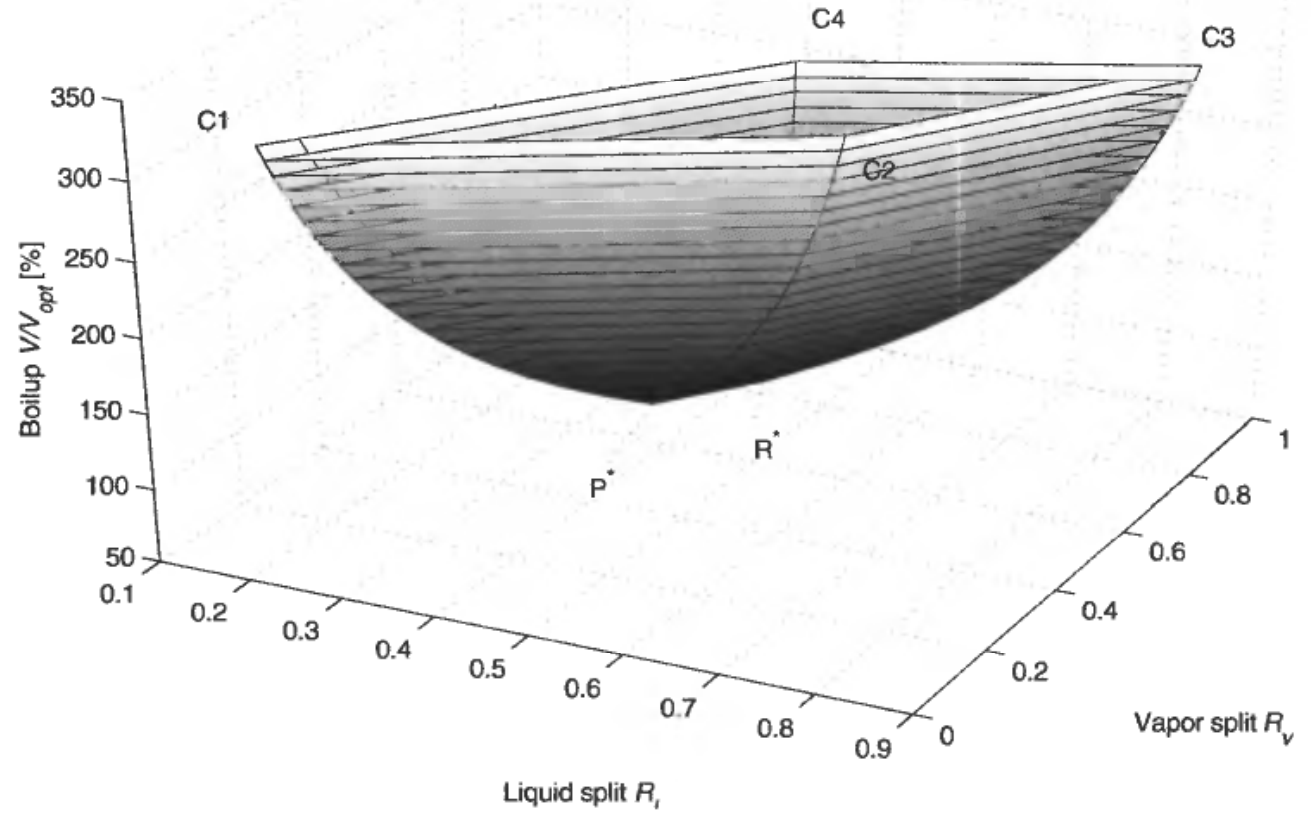

Figure 12. The solution surface $V\left(R_{l}, R_{v}\right)$ for the limiting case with infinite stages and sharp splits contains the same characteristics as found in Figure 6.

(C1-C4). These contour line corners $(\mathrm{Cl}-\mathrm{C} 4)$ are illustrated seen in Figure 13 and each represent a particular operating condition for each particular edge (dotted) of the solution surface $V\left(R_{l}, R_{v}\right)$ :

Corner line $1(\mathrm{Cl})$ :

Preferred split in the prefractionator. Over-refluxed main column.

$\beta=\beta_{P}, L_{1}=L_{1, P}, L>L_{\min }$

Corner line $2(\mathrm{C} 2)$ :

Along the left branch of the minimum reflux characteristics for the prefractionator.

$\beta<\beta_{P}, L_{1}=L_{1, \min }(\beta), L=L_{\min }\left(\beta, L_{1}\right)$

Corner line $3(\mathrm{C} 3)$ :

Over-refluxed prefractionator (above the V-shaped minimum curve). Balanced main column.

$\beta=\beta_{R}(L), L_{1}=L_{1, R}(L), L>L_{\text {min }}\left(L_{1}>L_{1, \min }\left(\beta_{R}\right)\right)$

Corner line $4(\mathrm{C} 4)$ :

Along the right branch of the minimum reflux characteristics for the prefractionator, but above the point representing a balanced main column.

$\beta>\beta_{R}, L_{1}=L_{1, \min }(\beta), L=L_{\min }\left(\beta, L_{1}\right)$

Note that line $\mathrm{C} 2$ and $\mathrm{C} 4$ apply for our example where $\beta_{P}<\beta_{R}$. When $\beta_{P}>\beta_{R}$ we instead get the similar lines $\mathrm{C2}^{\prime}$ and $\mathrm{C4}^{\prime}$ : 


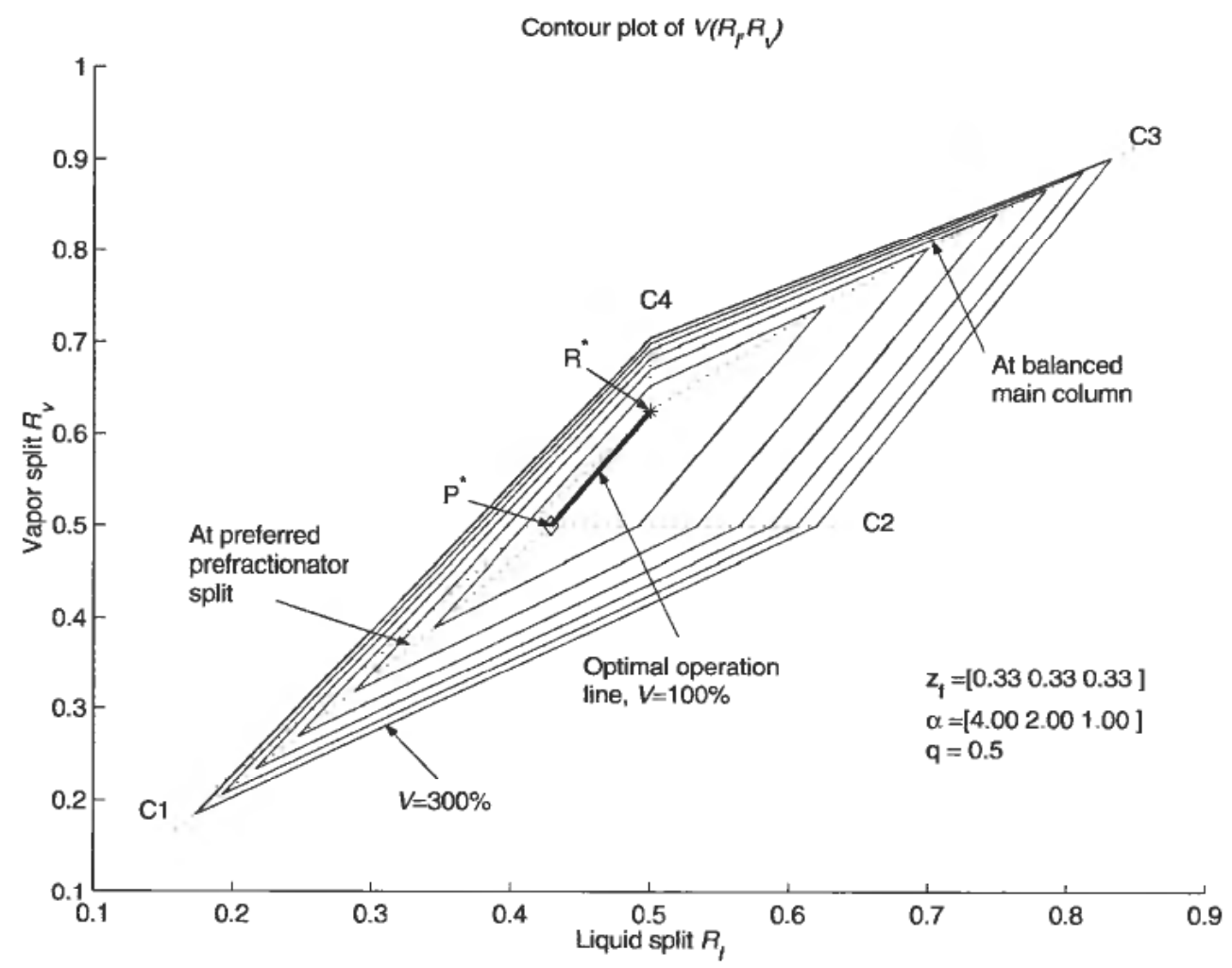

Figure 13. The contour lines for $V\left(R_{l}, R_{v}\right)$ are straight lines between the four characteristic corners. (The "bad" direction is normal to $\mathrm{P}^{*} \mathrm{R}^{*}$.) Contour plot of $V$ corresponding to Figure 12.

Corner line $2^{\prime}\left(\mathrm{C} 2^{\prime}\right)$ :

Along the right branch of the minimum reflux characteristics for the prefractionator.

$\beta>\beta_{P}, L_{1}=L_{1, \min }(\beta), L=L_{\min }\left(\beta, L_{1}\right)$

Corner line $4^{\prime}\left(\mathrm{C4}^{\prime}\right)$ :

Along the left branch of the minimum reflux characteristics for the prefractionator.

Above the point representing a balanced main column.

$\beta<\beta_{R}, L_{1}=L_{1, \min }(\beta), L=L_{\text {min }}\left(\beta, L_{1}\right)$

As we approach minimum boilup $\left(V=V_{\min }\right)$, lines $\mathrm{C} 1$ and $\mathrm{C} 2$ (or $\left.\mathrm{C} 2^{\prime}\right)$ approach point $\mathrm{P}^{*}$ (optimum at preferred prefractionator split, $\beta=\beta_{P}$ ) and line $\mathrm{C} 3$ and $\mathrm{C} 4$ (or $C 4^{\prime}$ ) approach point $\mathrm{R}^{*}$ (optimum at balanced main column, $\beta=\beta_{R}$ ).

The path $\mathrm{C} 2-\mathrm{P}^{*}-\mathrm{R}^{*}-\mathrm{C} 4$ on the solution surface $V\left(R_{l}, R_{v}\right)$ represent an important limiting case of operating conditions: there the minimum reflux constraints are met in both the prefractionator and in the main column. That is: $L_{1}=L_{1 \cdot \min }(\beta)$ and $L=L_{\min }\left(\beta, L_{1}\right)=L_{\min }(\beta)$.

In the whole operating region to the right of the path $\mathrm{C} 2-\mathrm{P}^{*}-\mathrm{R}^{*}-\mathrm{C} 4$ in Figure 13 we over-reflux the prefractionator (operating above the V-shaped minimum characteristics), while we keep the main column at its minimum reflux: $L_{1}>L_{1, \min }(\beta)$ and $L=L_{\min }\left(\beta, L_{1}\right)$. This part corresponds to surfaces in the $\left(\beta, L_{1}\right)$-plane found in 
Fidkowski and Krolikowski (1986). Note also that the case of a balanced main column is always within this region (along $\mathrm{C} 3$ ).

In the whole operating region to the left of the path $\mathrm{C} 2-\mathrm{P}^{*}-\mathrm{R}^{*}-\mathrm{C} 4$ in Figure 13 we operate the prefractionator exactly at its minimum characteristic $\left(L_{1}=L_{1, \min }(\beta)\right)$, but we over-reflux the main column $L>L_{\min }\left(\beta, L_{1}\right)$. The computation of the surface in this region is a new contribution as it was not considered by Fidkowski and Krolikowski (1986).

Finally, we must note that the "good direction" is along the path $\mathrm{Cl}-\mathrm{P}^{*}-\mathrm{R}^{*}-\mathrm{C} 3$ (which is coinciding with the path $\mathrm{C} 2-\mathrm{P}^{*}-\mathrm{R}^{*}-\mathrm{C} 4$ only along the line $\mathrm{P}^{*} \mathrm{R}^{*}$ ). Operation along the "good" path gives the minimum of $V$ when we keep one degree of freedom constant $\left(R_{l}\right.$ or $\left.R_{v}\right)$. Observe that $\mathrm{C} 1$ is to the left of the path $\mathrm{C} 2-\mathrm{P}^{*}-\mathrm{R}^{*}-$ $\mathrm{C} 4$ and $\mathrm{C} 3$ is to the right.

\subsection{Analyzing the effect of the feed enthalpy}

The effect of changing the liquid fraction is shown in Figure 14 (contour plot) and Figure 15 (cross section in the bad direction) for the infinite stage model.

The results in Figure 15 are in agreement with similar computations for the finite column model in Figure 8. As we increase $q$ the surface between corner lines $\mathrm{C} 4$ and $\mathrm{C} 1$ first becomes vertical and then starts to bend over when we increase the liquid fraction past saturated liquid $q \approx 1$.

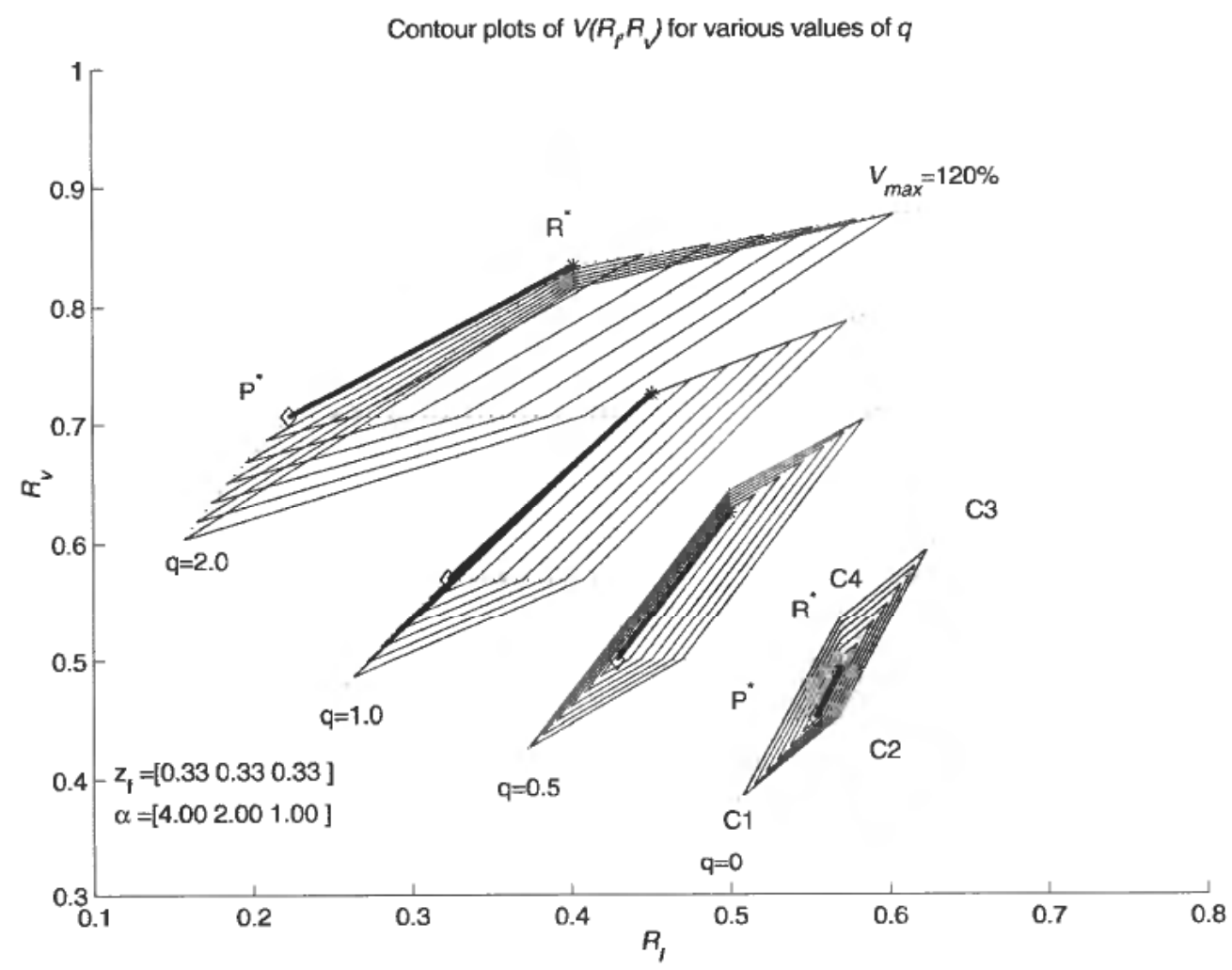

Figure 14. Liquid fraction affects the shape of the surface in addition to the position of the optimal operating line in the $V\left(R_{l}, R_{v}\right)$ plane. 


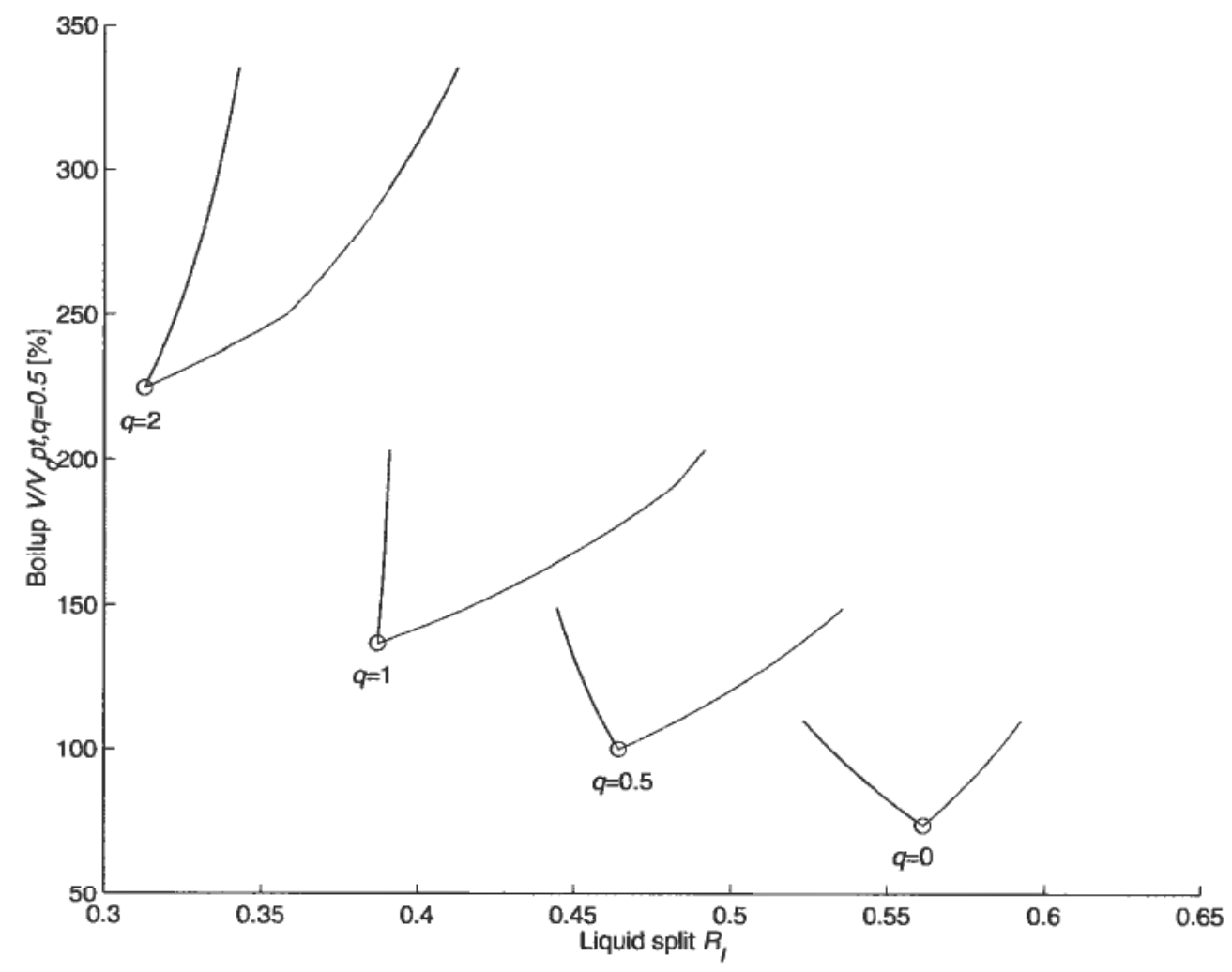

Figure 15. Cross-sections of the surfaces in Figure 14 in the "bad" direction normal to P*R* (taken at the middle of the line $\mathrm{P}^{*} \mathrm{R}^{*}$ ).

\subsection{How many degrees of freedom must be adjust during operation?}

Is it possible to obtain reasonable energy savings if we keep both $R_{v}$ and $R_{l}$ constant? The answer is clearly "no" for our case study, as we have already found that the energy usage (boilup $V$ ) increases very sharply as we move away in certain directions from the flat region. This is further illustrated in Figure 16, where we show the boilup as a function of $R_{l}$ for various fixed values of $R_{v}$ (this is not quite as bad as we move normal to $\mathrm{P}^{*} \mathrm{R}$, but note the difference in axis scaling when comparing the curve for $q=0.5$ in Figure 15 with Figure 16). We clearly see from the sharp minimum of the V-shaped curves (solid lines) that $R_{l}$ would have to be determined very accurately in order to obtain a value of $V$ reasonably close to the minimum. For instance, if $R_{l}$ is set only 5\% away from its optimal value, energy increase compared to the optimum is between $10 \%$ to $30 \%$.

Having established that we cannot keep both degrees of freedom constant, we ask: can we leave one constant? Since the vapour flows are usually the most difficult to adjust in practice, and since it seems reasonable in many cases that the vapor split is constant if we do no adjustments, we will analyze what happens when we keep $R_{v}=$ constant and then adjust the other degree of freedom (e.g. $\left.R_{l}\right)$ optimally.

Figure 17 shows how the boilup $(V)$ depends on $R_{v}$ when $R_{l}$ is optimized for every value of $R_{v}$ (i.e. along the "good" $\mathrm{Cl}-\mathrm{P}^{*}-\mathrm{R}^{*}-\mathrm{C} 3$ path in Figure 12). As mentioned above, we must choose $R_{v}$ in the flat region $\left(R_{v, p}<R_{v}<R_{v, r}\right)$ in order to achieve minimum boilup. Importantly, if $R_{v}<R_{v, p}$ or $R_{v}>R_{v, r}$ we very soon lose 


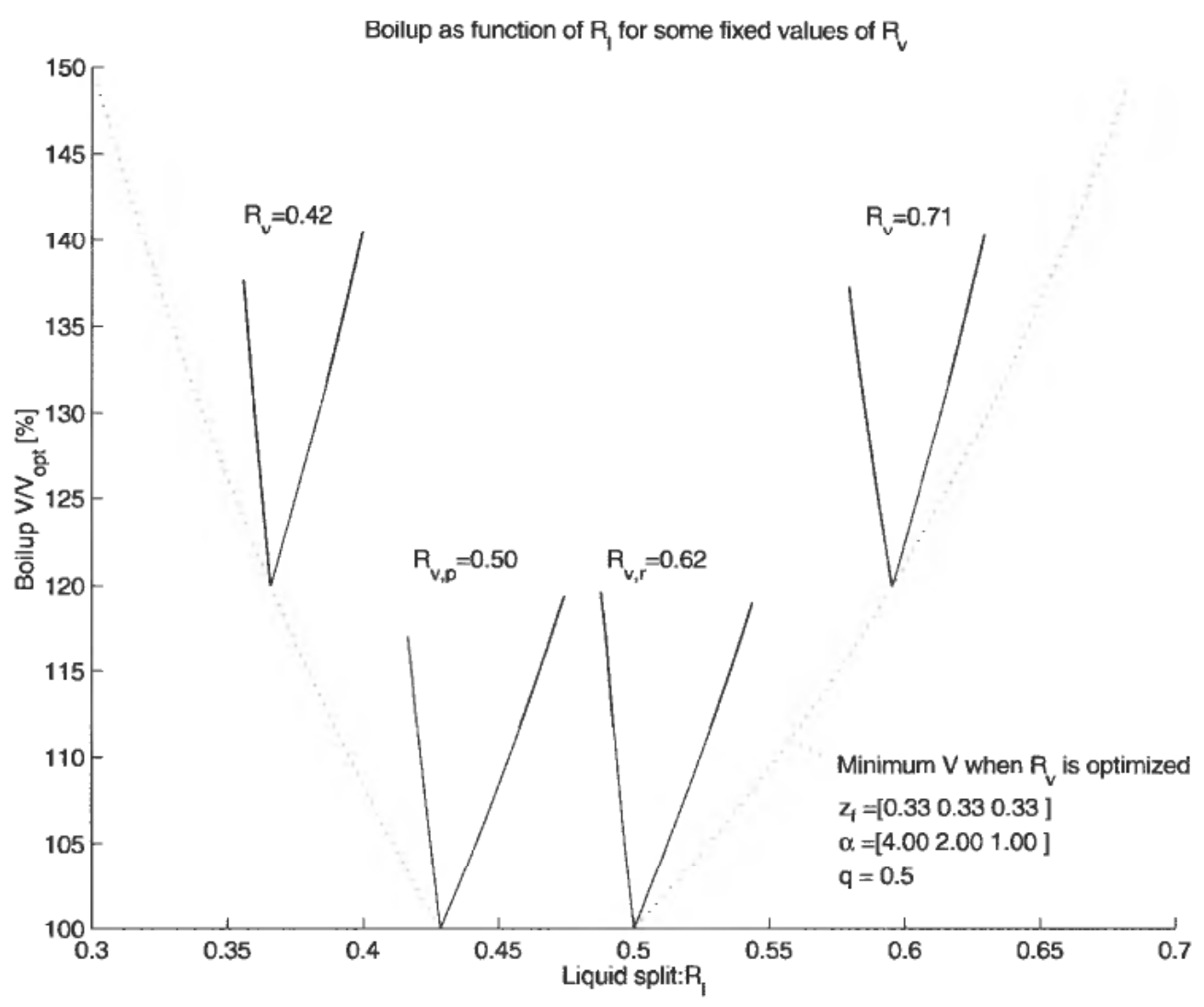

Figure 16. We have to adjust the liquid split on line in order to operate on minimum energy consumption.

energy compared to the optimal operation $\left(V>V_{\min }=100 \%\right)$ even if $R_{l}$ is adjusted optimally. For $R_{v}<R_{v, p}$, the best we can do is to adjust $R_{l}$ to operate the prefractionator exactly at its preferred split and minimum reflux, while the main column is over-refluxed (along $\mathrm{C} 1$ ). And for $R_{v}>R_{v, r}$ the best we can do is to adjust $R_{1}$ to operate the main column at the balance line, while the prefractionator is over-refluxed (along C3).

Also recall from Figure 16 that even with $R_{v}$ in the flat region, we will need to adjust $R_{l}$. We conclude that it is acceptable to keep one degree of freedom (e.g. $R_{v}$ ) constant, as long as it is selected so to operate within the flat region, and as long as the other degree of freedom is adjusted optimally.

\subsection{Sensitivity to disturbances and model parameters}

We want to check if the simple strategy of keeping $R_{v}$ constant will work. In Figure 18 we show the set of "flat region" (minimum energy) line segments $\left(\mathrm{P}^{*} \mathrm{R} *\right)$ for variations of feed enthalpy $(q=[0 \cdot 40 \cdot 50 \cdot 6])$ and $2 \%$ feed composition changes in different directions $\left[z_{a}, z_{b}\right]=[1 / 3,1 / 3]+0 \cdot 02[\cos (\gamma), \sin (\gamma)], \gamma=\left[0,30^{\circ}, \ldots, 360^{\circ}\right]$.

When the light feed fraction is increased and the heavy reduced, the points $\mathrm{P}^{*}$ and $\mathrm{R}^{*}$ move closer together, reducing the flat region. Changes in $q$ result in sideways movement of the $\mathrm{P}^{*} \mathrm{R}^{*}$ linc. The possible region for $R_{v}$ that ensures operation in the 


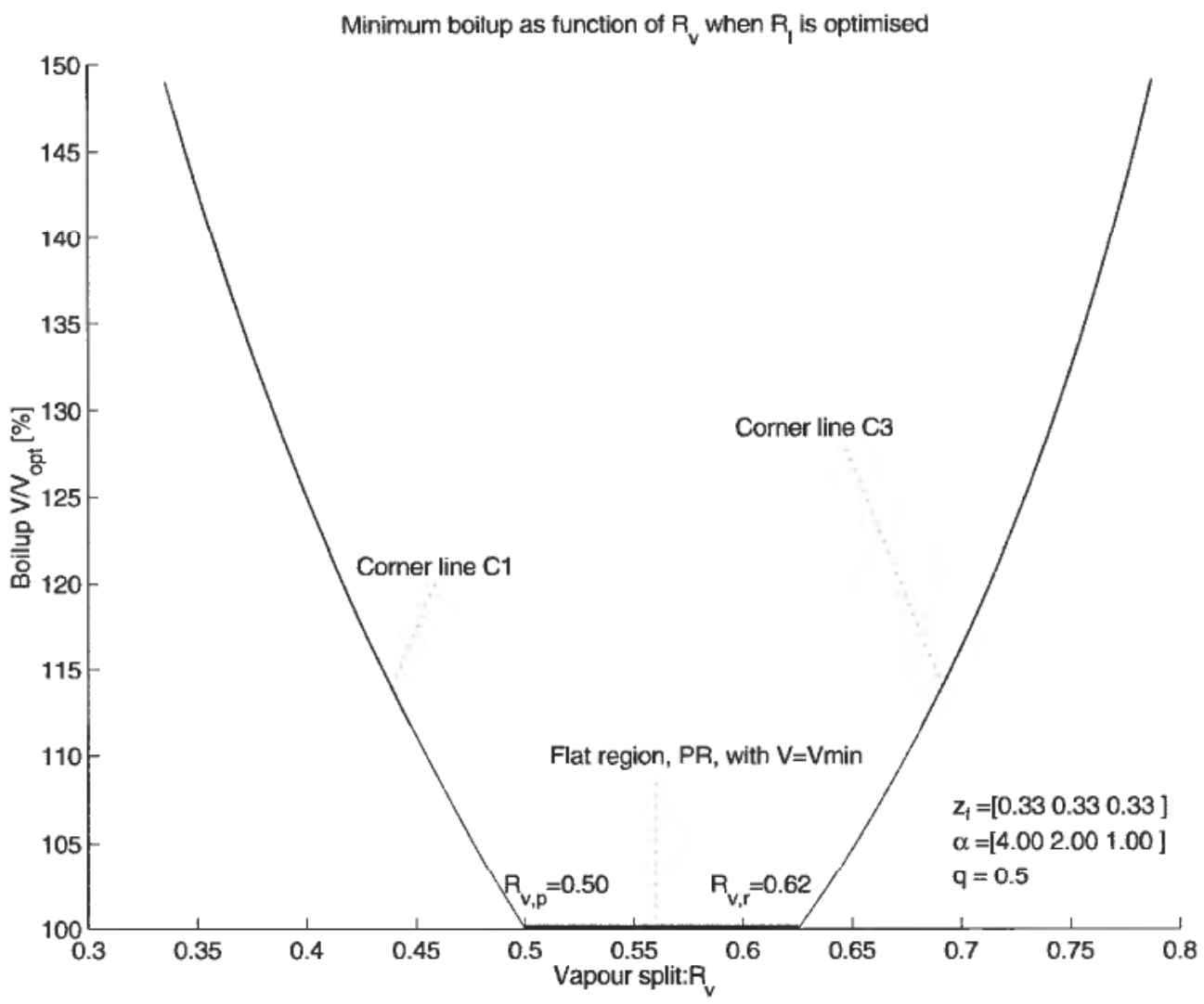

Figure 17. Minimum energy can be obtained if the vapor split is set within the flat region. Plot show $V$ as a function of $R_{v}$ when $R_{l}$ is optimized for each value of $R_{v}$.

flat region for all possible disturbances in our example is indicated by the quite narrow region between the solid and dashed lines.

\subsection{A simple control strategy with one degree of freedom fixed}

Based on the observations above we propose a control strategy where we fix $R_{v}$ and use $R_{l}$ as a manipulated input. (We could also make the opposite choice.)

1. Keep a fixed value for $R_{v}$ in the flat region $R_{v} \in\left[R_{v, p}, R_{v, r}\right]$

2. Control the product compositions at their setpoints (e.g. by manipulating $L$, $S$ and $V$ ).

3. Control some feedback variable such that $R_{l}$ is being adjusted close to optimally.

Provided that we can find the right feedback variable, this strategy will be acceptable if the magnitude of feed disturbances and other uncertainties do not bring the selected $R_{v}$ outside the flat region. If the latter is not satisfied, we will hgave to adjust also $R_{v}$ to keep the operation within the flat region.

A particular difficult case occurs if some disturbance moves the balance point for the main column to the other wise of the point of preferred split. In this case $R_{v}$ will usually have to be adjusted, and we may have to change the control strategy for adjusting $R_{l}$. 


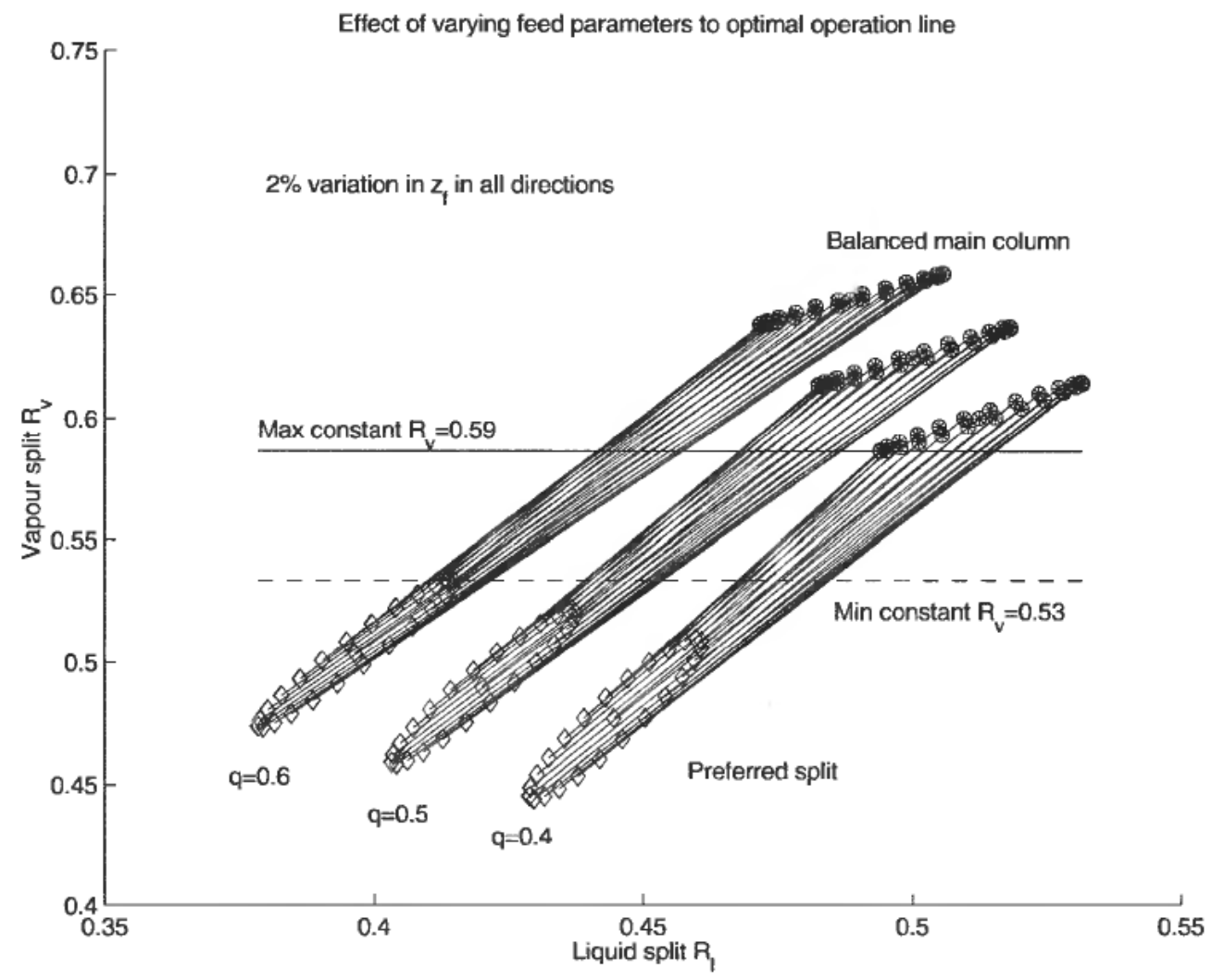

Figure 18. For the selected set of disturbances, a value of $R_{v}$ between 0.53 and 0.59 guarantees operation in the flat region.

\subsection{Liquid fraction: bad disturbance or extra degree of freedom?}

In general, adding more heat in the feet (i.e. reducing liquid fraction $q$ ) will be less efficient than adding the same heat in the reboiler. However, recall from Figure 15 that the position of the minimum energy line $\left(\mathrm{P}^{*} \mathrm{R}^{*}\right)$ will be directly affected by the feed enthalpy and this may be used to our advantage. For instance, in a case where we cannot adjust $R_{v}$, and we are operating outside the "flat" minimum energy region, we may add heat or cool the feed to move the solution surface into the flat region. Flow constraints in the column sections may be another motivation for introducing the feed enthalpy as a degree of freedom.

It is also possible to introduce an extra degree of freedom by extracting both liquid and vapour products in the sidestream, again for the purpose of moving the solution surface as desired.

In summary, large uncontrolled variations in the liquid fraction should be avoided, but adjustments of the feed enthalpy $(q)$ can be used as a mean to move the solution surface in a desired manner.

\subsection{Relations to composition profiles}

Each of the different surface segments in Figure 12 corresponds to a characteristic composition profile. The location of the pinch zones on these profiles can be used to 

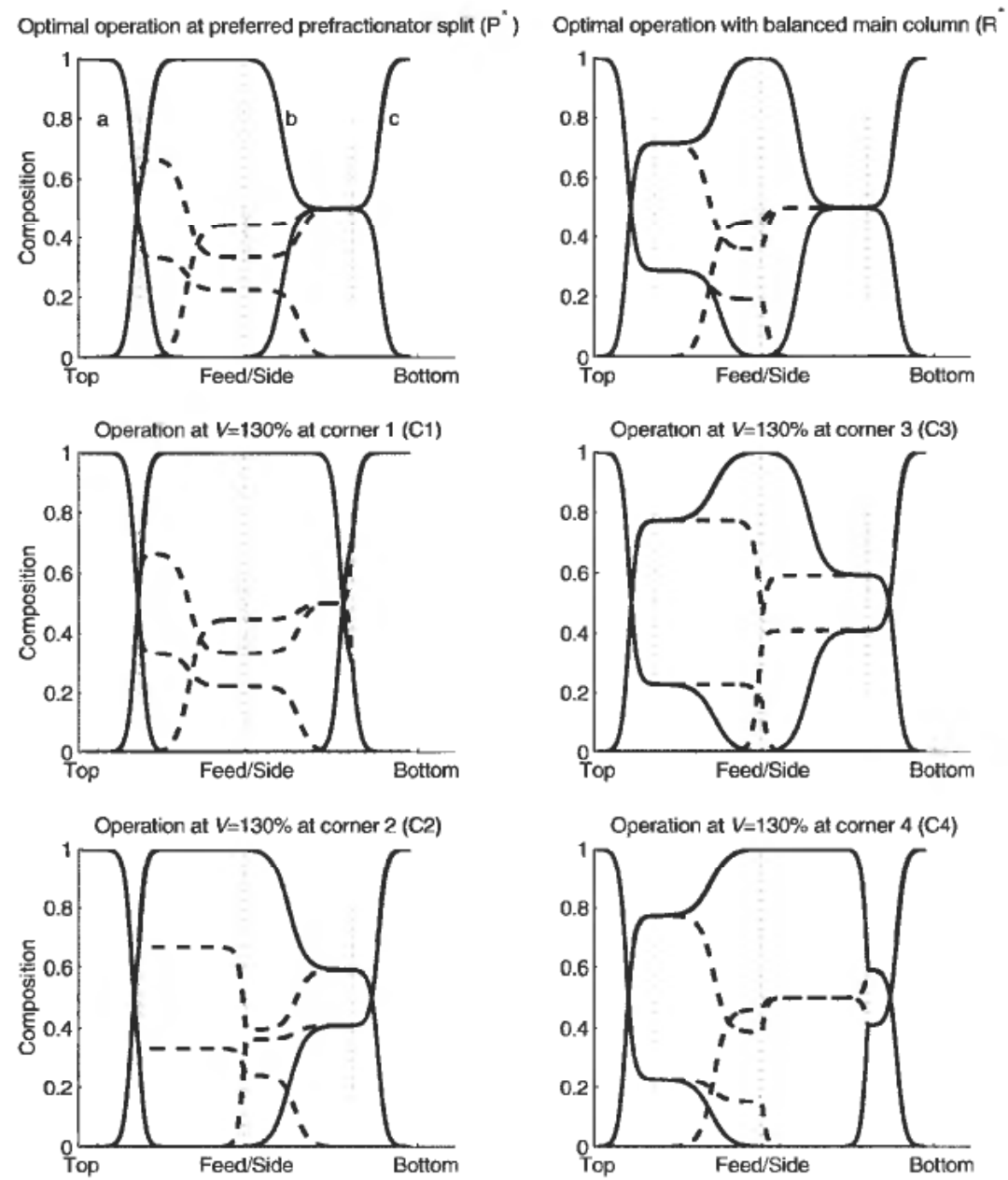

Figure 19. We can extract important information about the actual operating point from the composition profiles in the "infinite" Petlyuk column. Prefractionator composition profiles are shown dashed. Locations of feed, sidestream and connection stages are indicated (dotted). Feed data: $z f=[0 \cdot 33,0 \cdot 33,0 \cdot 33], \alpha=[4,2,1], q=0 \cdot 5$.

identify the actual operation point, and this information may then be used in an optimizing control strategy. In Figure 19 we show composition profiles computed from the stage-by-stage column model with a sufficiently large number of stages to be a good approximation of an infinite column. (Adding more stages will just extend the flat pinch regions.) We show composition profiles for 6 different operating points: optimal operation $\left(V=V_{\min }\right)$ at $\mathrm{P}^{*}$ (upper left) and $\mathrm{R}^{*}$ (upper right), and suboptimal operation $\left(V=1.3 V_{\min }\right)$ along the four corner lines $\mathrm{Cl}$ to $\mathrm{C} 4$. We used the infinite stage model to compute the control inputs for each case (e.g. Figure 13).

At operating point $\mathrm{P}^{*}$ we have pinch zones on both sides of the prefractionator feed, and at the lower "feed" to the main column, whereas the upper part of the main column is over-refluxed. At point $\mathrm{R}^{*}$ we have pinch zones at both "feeds" to the main column (the column is balanced), but here the lower end of the prefractionator is over-refluxed. (Remember that we have $\beta_{P}<\beta_{R}$, and in the case of $\beta_{P}>\beta_{R}$ we would get an anti-symmetric result.) Along $\mathrm{C} 1$ (middle left) we have a similar prefractionator 
profile as at $\mathrm{P}^{*}$, but along $\mathrm{Cl}$ both parts of the main column is over-refluxed. And similarly, along C3 (middle right) the main column is balanced at minimum reflux (like in $\mathrm{R}^{*}$ ), whereas the prefractionator is over-refluxed along C3. Along C2 (lower left) we over-purify the "wrong" (upper) side of the prefractionator, and along C4 (lower right) we over-reflux the "wrong" (lower) end of the main column.

The optimal "pattern" in our case study, where $\beta_{P}<\beta_{R}$, is to have a pinch zone above the prefractionator feed, and a pinch zone on both sides of the lower main column "feed". If this is the case, we know that the operation is along line $\mathrm{P}^{*} \mathrm{R}$ *. None of the suboptimal operating points have this "signature". Note also that for operation along $\mathrm{P}^{*} \mathrm{R}^{*}$, the upper part of the main column and the lower end of the prefractionator, are over-refluxed. In cases with $\beta_{P}<\beta_{R}$ both pinch zones move to the other end. If we do not know the relative magnitude of $\beta_{P}$ and $\beta_{R}$, a possible approach is to operate at point $\mathrm{P}^{*}$ all the time, that is, with pinch zones of both sides of the prefractionator feed (or no end of the prefractionator overpurified).

The corresponding column with a finite number of stages and non-sharp splits studied earlier (Table 1 and Figure 4) does not have pinch zones, and this tells us that we probably have too few stages. However, that model is not intended as a column design example, but rather to illustrate the problem of optimizing control. And more importantly, in spite of low number of stages in our case study example, the main properties of that solution surface is very close to the results from the infinite stage model.

\section{Candidate feedback variables}

The results from computations using models with both finite and infinite number of stages show that we must continuously adjust at least one of the two degrees of freedom (e.g. $\left.R_{l}\right)$ if close to optimal operation is desired. As mentioned above, we would like to implement this in a feedback fashion, by finding some measurement, which when kept at a constant value, indirectly ensure optimal operation. Candidates for such measurements are composition measurements on individual stages, temperature measurements and combinations thereof and flow measurements from individual sections of the column. Temperatures are easy to measure, flows are more difficult, and even more so are compositions.

We consider next a few candidate measurements (Y1-Y6) for feedback control. The analysis is mainly based on observations from the model with a finite number of stages.

\subsection{Position of profile in main column (Y1)}

An interesting observation from our case study using the finite stage model is that the maximum composition of the mid-component occurs at the location of the side-stream when the column is at its optimum (Figure $4 \mathrm{~b}$ ). A measurement of the stage number with the maximum value of the intermediate component $x_{b}$ therefore seems to be a very good candidate for feedback optimization. However, we would need on-line composition measurements on several stages, so it is difficult to use in practice.

\subsection{Temperature profile symmetry (Y2)}

The temperature profiles on both sides of the dividing wall show some interesting symmetry properties. We define the average difference temperature of the temperature 

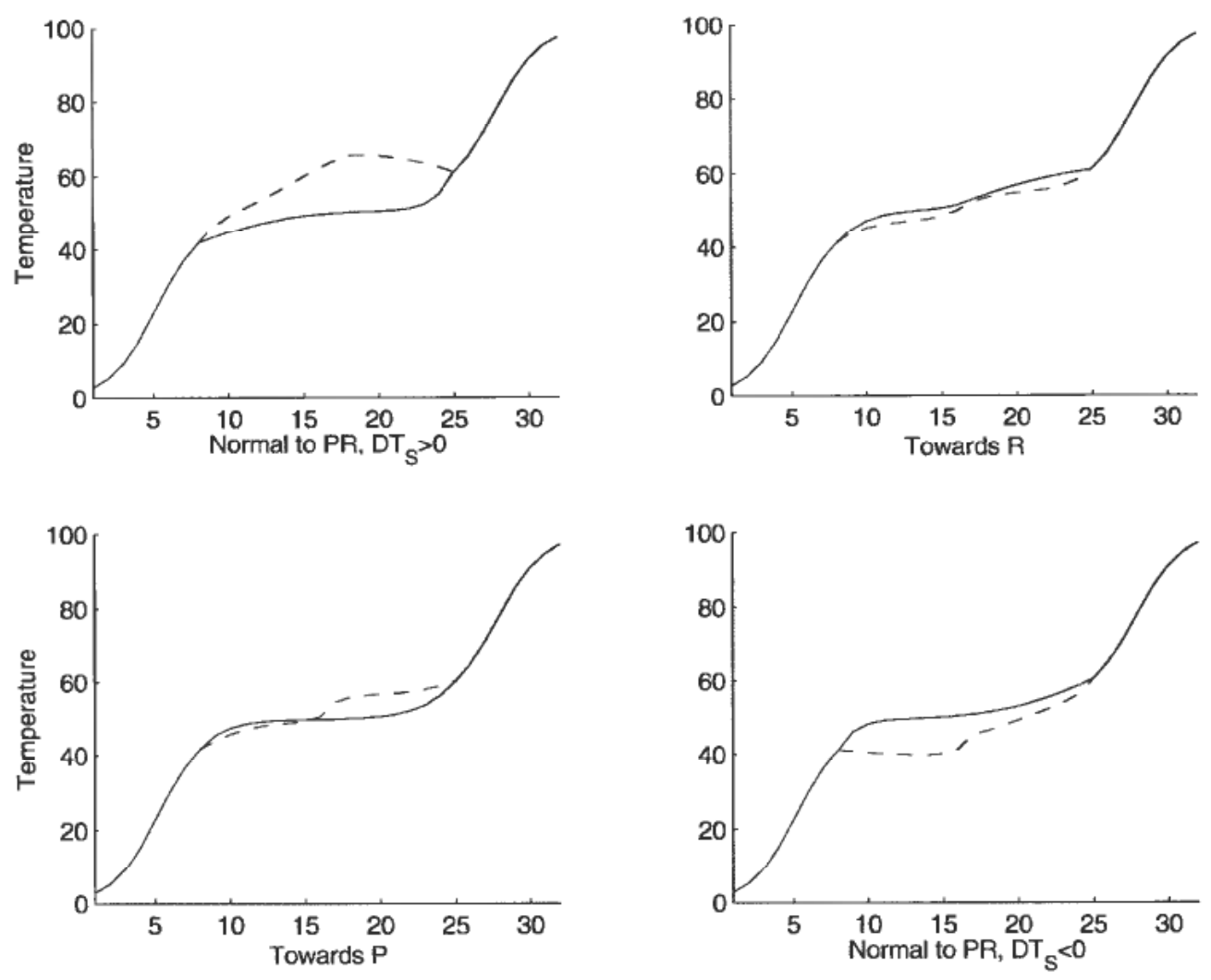

Figure 20. Temperature profiles for the base case for off-optimal operation in directions along the bottom valley towards $\mathrm{R}$ (upper right) and $\mathrm{P}$ (lower left), and in the "bad" directions normal to PR to the left (upper left plot) and to the right (lower right plot).

profiles on each side of the dividing wall as a symmetry measurement $\left(D T_{S}\right)$. If the vector $T_{p, k}$ contains the temperature profile in section $k$, and $\bar{x}$ denotes the average of the elements in the vector $\mathrm{x}$, then

$$
D T_{S}=\left(\overline{T_{p, 1}}-\overline{T_{p, 4}}\right)+\left(\overline{T_{p, 2}}-\overline{T_{p, 5}}\right)
$$

In a practical application $D T_{S}$ can be based on one or more pairs of difference temperatures in sections above and below feed and side stream. The temperature profile shown in Figure 5 is for optimal operation. In Figure 20 we show the profiles if we move away from the optimum in the four directions towards $P$ and $R$ and normal to PR in Figure 6. Interestingly we find that $D T_{S}$ is close to constant along directions parallel to the "bottom of the valley" of the solution surface (along PR in Figure 6), as illustrated in Figure 21. When we move away from the bottom of the valley normal to $\mathrm{PR}$, the profile symmetry changes, and the $D T_{S}$ becomes more positive towards the right side and more negative to the left side of PR (see Figure 7).

If we choose to adjust the liquid split $\left(R_{l}\right)$ to control $D T_{S}$, we can replace the liquid fraction $\left(R_{l}\right)$ with the setpoint for $D T_{S}$ as a degree of freedom. The contour plot of the surface $V\left(D T_{S}, R_{v}\right)$ for the base case is shown in Figure 22 and when we compare this to the contour of $V\left(R_{l}, R_{v}\right)$ in Figure 7 we observe that the region close to the optimum now is quite flat in both directions of the degrees of freedom for $V\left(D T_{D}, R_{v}\right)$ as opposed to $V\left(R_{l}, R_{v}\right)$ which is quite steep in the dircction normal to 


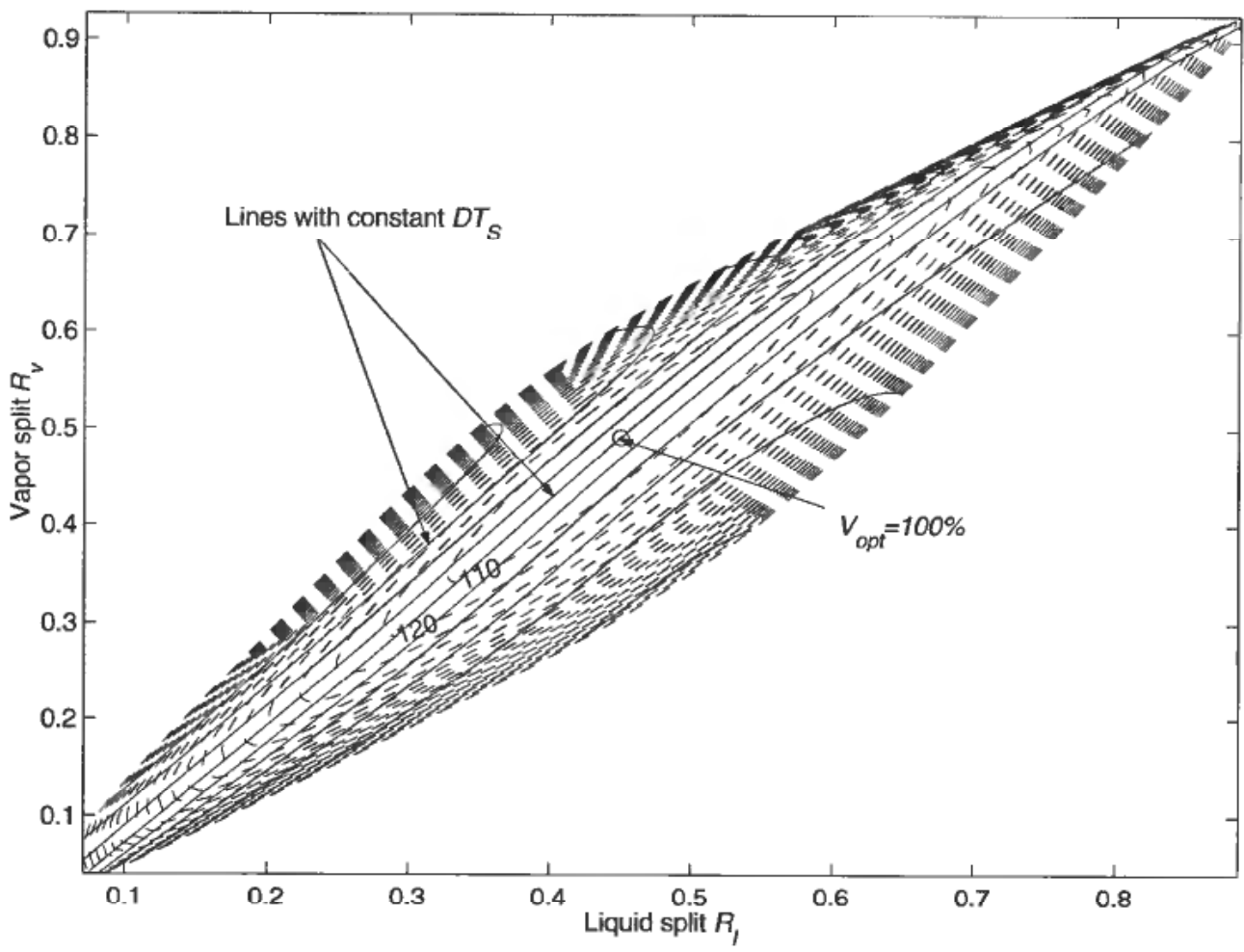

Figure 21. Operation at constant $D T_{S}$ implies operation at a line parallel to the good PR direction on the solution surface. Plot show contour lines of constant $D T_{S}$ (solid) projected on the contour lines of $V\left(R_{l}, R_{v}\right)$ (dashed) for the base case in Table 1 .

line PR. This "flatness" is a very important property since it implies that the energy consumption will not be very sensitive to the degrees of freedom in the flat region.

Unfortunately, the optimal value of $D T_{S}$ (which may be non-zero), is sensitive to feed composition disturbances. However, $D T_{S}$ is easy to measure and apply in a practical control strategy.

\subsection{Impurity of prefractionator output flows (Y3, Y4)}

A key to optimal operation is to operate the prefractionator at minimum reflux characteristic $L_{1}=L_{1, \min }(\beta)$. Christiansen and Skogestad (1997) showed that this is achieved by:

1. $\beta>\beta_{P}$ : Control the impurity of the heavy component in the top. (Y3)

2. $\beta<\beta_{P}$ : Control the impurity of the light component in the bottom. (Y4)

In both cases the uncontrolled end of the prefractionator should be over-purified.

In cases when $\beta_{P}$ and $\beta_{R}$ are close or may change order, we would have to use both degrees of freedom if we want to track the optimum. Since we know that operating the prefractionator at the preferred split always will be optimal, independent of where the balance point is, we can look for a strategy which keeps the prefractionator operating point at the preferred split all the time. $\left(L_{1, P}, \beta_{P}\right)$ This can be obtained by using both degrees of freedom for two-point control of both the prefractionator impurities ( $\mathrm{Y} 3$ and $\mathrm{Y} 4)$. 


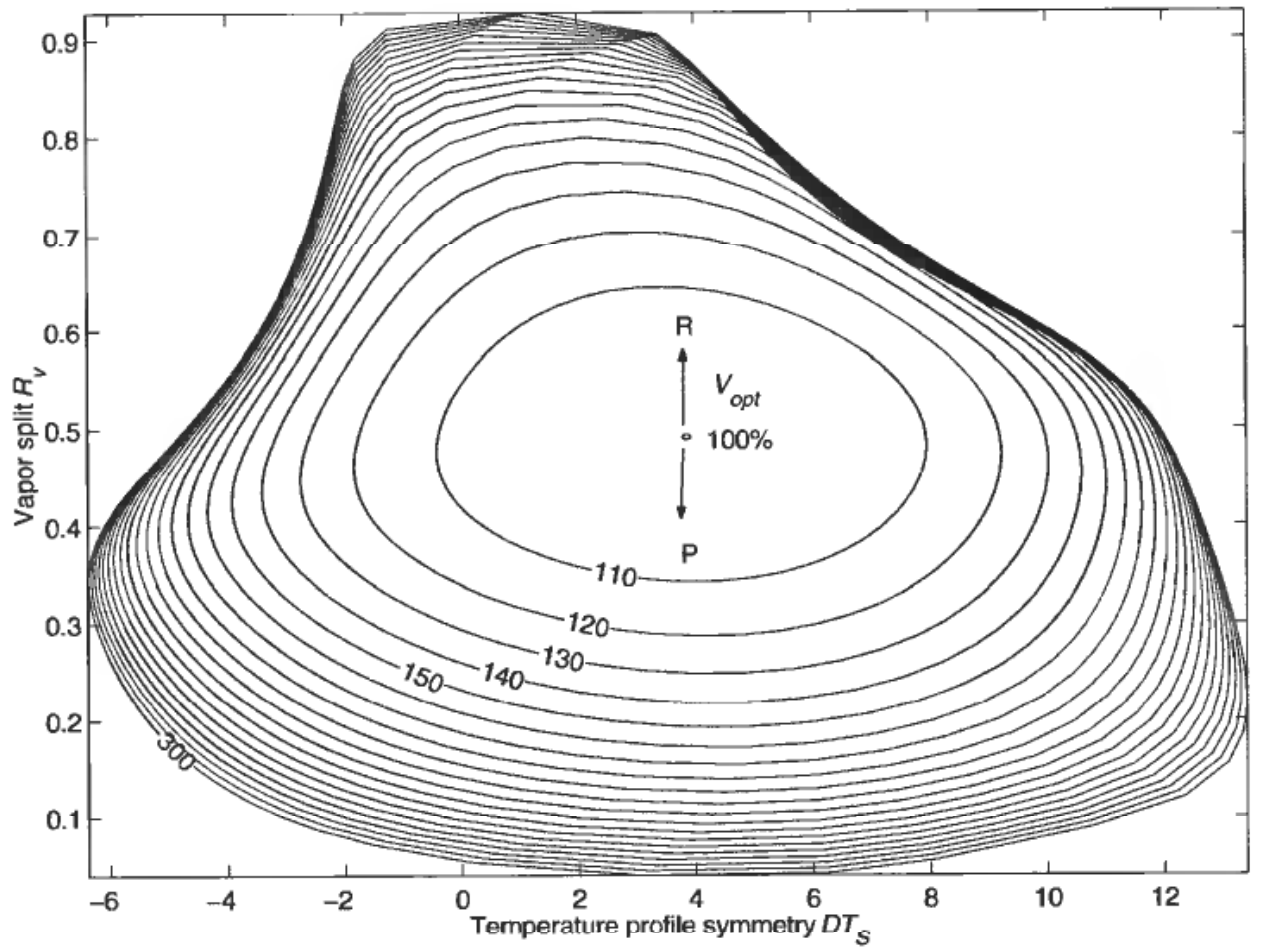

Figure 22. Contour plot of $V\left(D T_{S}, R_{v}\right)$ for the base case. The region close to the optimum is now quite flat in both directions.

We also have to ensure that the main column is operated at its minimum reflux. But this is indirectly achieved by controlling all three product purities.

\subsection{Prefractionator flow split (Y5)}

Consider the net "distillate" flow leaving the top of the prefractionator $\left(D_{1}\right)$.

$$
D_{1}=V_{1}-L_{1}
$$

Note that this is not a physical stream, but a difference between the vapor and liquid flows in the top of the prefractionator. It may even become negative if the column is not operated well. For sharp splits, $D_{1}=z_{a}+\beta z_{b}$ (for a normalized feed $F=1$ ) so by adjusting $D_{1}$ we directly affect the distribution of the middle component (b). We would expect $\beta$ to be in the range $[0,1]$, and thus $D_{1}$ to be in the range $\left[z_{a}, z_{a}+z_{b}\right]$. This insight is correct, as we find in some non-optimal operating points that $\beta$ or even $D_{1}$ may be negative, corresponding to circulation around the dividing wall. Boilup as a function of $D_{1}$ is illustrated in Figure 23, where we see that $D_{1}$ changes almost proportionally to the boilup when we move along the solution surface in the bad direction normal to PR. Thus if we were able to measure the net prefractionator distillate flow $D_{1}$, then we could achieve close to optimal operation by adjusting $R_{l}$ (or $L_{1}$ ) to keep $D_{1}$ at a setpoint. Unfortunately such a flow measurement is difficult to obtain in practice.

We can also express $D_{1}$ in terms of $R_{l}$ and $R_{v}$. A simple overall material balance for the prefractionator yields:

$$
D_{1}=R_{v} V-R_{l} L+(1-q)
$$




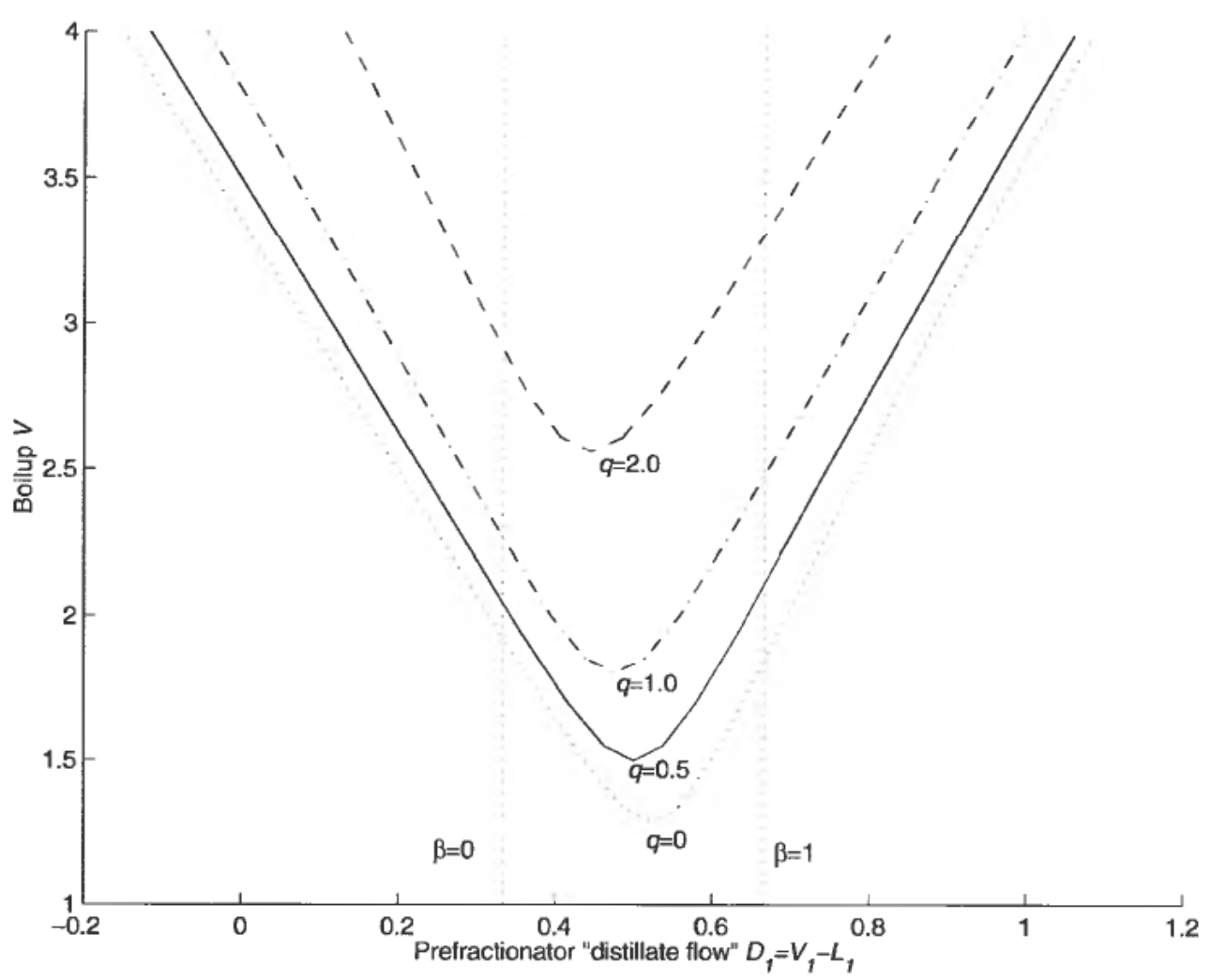

Figure 23. Boilup $V$ as function of the prefractionator "distillate" flow $\left(D_{1}\right)$ in the "bad" directional normal to PR.

where $L$ and $V$ are the overall reflux and boilup for the main column. This shows that $R_{v}, R_{l}$ and $q$ affects $D_{1}$ in a similar way.

Another very interesting observation is that $V$ as a function of $D_{1}$ behaves very "nicely" (Figure 23), compared to the very non-linear relationship between $V$ and $R_{l}$ (Figure 8) where we may even have multiple solutions in some cases. This shows that if we were to use an open-loop ppolicy, it would be better to keep $D_{1}$ rather than $R_{t}$ constant. For example, for $q=1$ we see upon comparing Figures 8 and 23 that a very small reduction in $R_{l}$ yields a large increase in $V$, since the surface $V\left(R_{l}, R_{v}\right)$ is very steep close to the optimum. On the other hand, From Figures 23 and 24 we observe that this is not the case with $D_{1}$ as an independent variable.

\subsection{Temperature difference over prefractionator (Y6)}

It is possible to find variables that have an extremal value when $V=V_{\min }$. Such variables cannot be used for feedback setpoint control approaches because the steadystate changes sign at the optimum. However, often it is difficult to directly measure the criterion value $(V)$. In such cases other variables may be used instead as an indicator of the criterion value and, used for example, in an on-line experimenting method (like EVOP).

One such variable is the temperature difference over the pre-fractionator (Y6). We observe from the model with a finite number of stages that the temperature difference 


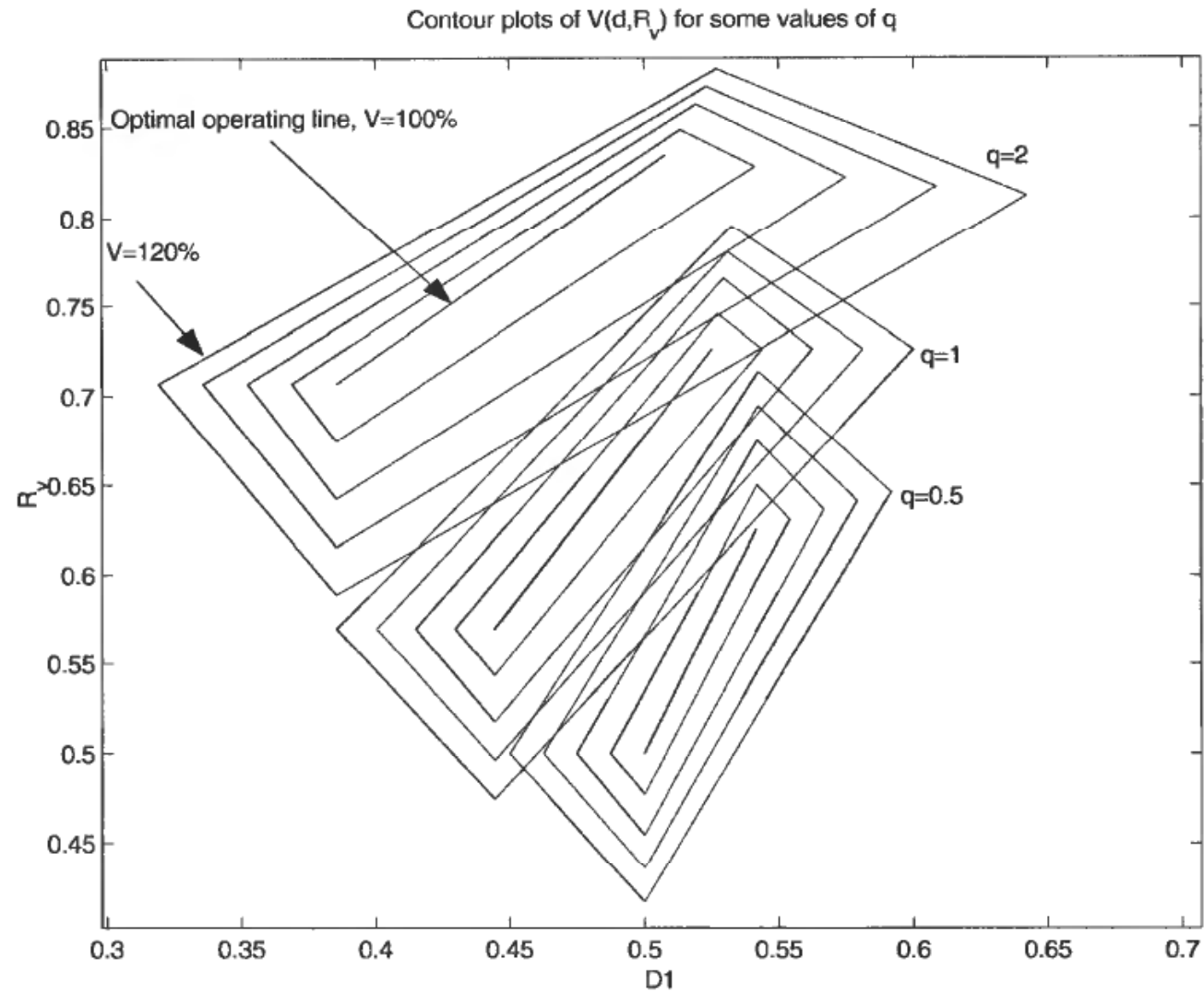

Figure 24. The surface $V\left(D_{1}, R_{v}\right)$ is less sensitive to variations in feed liquid fraction than the equivalent $V\left(R_{l}, R_{v}\right)$ when we fix both degrees of freedom. (Plots for model with infinite stages.)

over the pre-fractionator always has its maximum when the boilup is at its minimum. Although it is simple to measure, the actual maximum value depends on disturbances and product purities, so it may be difficult to tell the difference between the effect of non-optimal operation, or a disturbance, like changed feed composition.

\subsection{Evaluation of feedback candidates}

A qualitative evaluation of the various alternative measurements introduced above is shown in Figure 25. The criterion function is the boilup $V$ and in particular we need to avoid movement in the "bad" direction normal to PR. The position of the maximum $b$-composition in the main column is promising as a feedback variable since it, at least for our case study, is not affected by disturbances at all, but it may be difficult to measure or estimate. The other variables are affected by disturbances and setpoints, thus keeping one of these constant may lead to operation away from the optimum as illustrated in the figure.

Nevertheless, the improvement may be significant, compared to keeping for example $R_{l}$ at a constant value. Feedback from the impurity of the heavy key in the top of the prefractionator (Y3 or Y4) is very interesting, but in this case one or two composition measurements are probably required. 


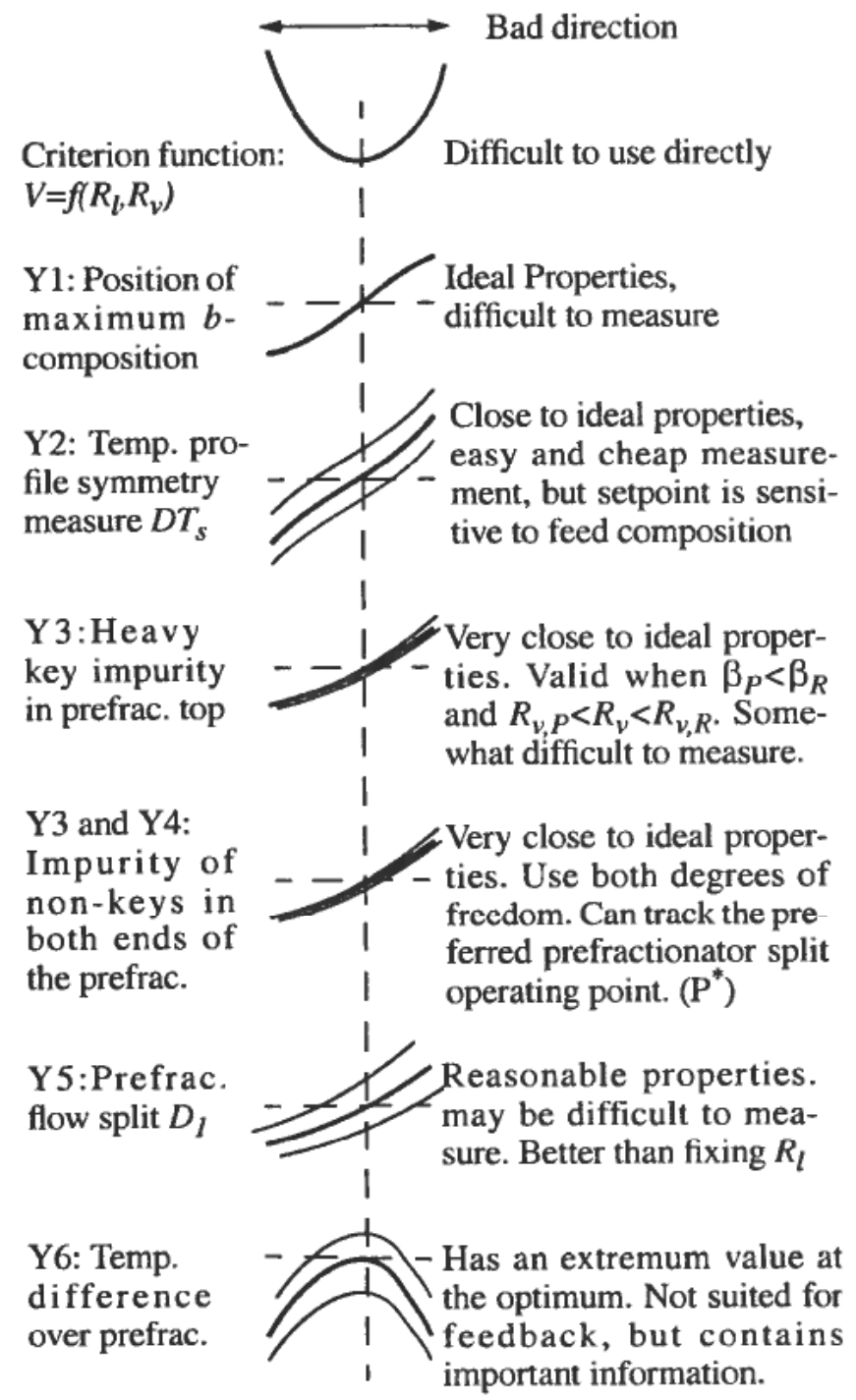

Figure 25. Some candidate feedback variables.

\section{Conclusions}

The Petlyuk distillation column will most likely require some kind of optimizing control in order to realize its full potential for reduced energy consumption. This is because the solution surface of the criterion function is very steep in one direction, and the operation is very sensitive to certain disturbances. The simplest strategy is to achieve "self-optimizing" control by feedback control of a variable which characterize optimal operation. In this paper we have obtained some relationships between optimal operation and some measurements which can be deduced from the composition profile or the states. This may be used to select candidate feedback variables. Optimization by feedback, or "self-optimizing control", should be compared to nonlinear model-based optimization methods, and evaluated for complexity and performance. 


\section{Acknowledgements}

This study has been founded in part by the European Community through the JOULE III programme within the project: "Complex Distillation Columns" (DISC), Contract no JOU-3-CT95-0035.

\section{Appendix A}

\section{A.1. Model equations for the finite dynamic model}

The model equations are quite standard and are described below. The component mass balance on a stage $i$ (counting from the top) for components $j \in[a, b, c]$ is given by:

$$
L_{i-1}\left(x_{i-1, j}-x_{i, j}\right)+V_{i+1}\left(y_{i+1, j}-y_{i, j}\right)=0
$$

With constant relative volatility, the equilibrium is given by:

$$
y_{i, j}=\frac{\alpha_{j} x_{i, j}}{\sum_{j} \alpha_{j} x_{i, j}}
$$

The column is modeled by connecting the stages, and sections as shown in Figure 1. We assume constant molar flows, thus $V_{i}=V_{i+1}$ and $L_{i}=L_{i-1}$ inside a section and $M_{i}=$ const. The liquid and vapor splits are assumed to be realized by splitting the flows at two specified ratios. (Note that indices 1-6 here denote the 6 column sections)

$$
\begin{aligned}
& L_{1}=R_{l} L_{3} \\
& V_{2}=R_{v} V_{6}
\end{aligned}
$$

The practical implementation of liquid split and side-stream withdrawal may involve full withdrawal of all downcomer flow into an external accumulator, and controlled flow back into the column again. The vapor split may be more difficult to implement in practice, but practical solutions do exist.

The feed enthalpy factor is given in terms of the liquid fraction $q$ :

$$
\begin{array}{ll}
q>1 & \text { Subcooled liquid } \\
q=1 & \text { Saturated liquid } \\
0<q<1 & \text { Liquid and vapor } \\
q=0 & \text { Saturated vapor } \\
q<0 & \text { Superheated vapor }
\end{array}
$$

More precisely, the flow changes at the feed stage $(i=f)$ are given by:

$$
\begin{aligned}
L_{i-1} & =L_{i}+q F \\
V_{i} & =V_{i+1}+(1-q) F
\end{aligned}
$$

and the following expression is added to the component mass balance in equation (A.1) at $i=f$.

$$
q F\left(z_{j}-x_{i, j}\right)+(1-q) F\left(z_{j}-y_{i, j}\right)
$$

A simple temperature model is used here: we just assume that the temperature on a stage $(i)$ is the mole fraction average of the boiling points $T_{B, j}$ for each components $(j)$.

$$
T_{i}=\sum_{j=a, b, c} T_{B j} x_{i, j}
$$




\section{A.2. Analytic expressions for minimum reflux of an infinite Petlyuk column} (Halvorsen and Skogestad, 1999)

These results are based on Fidkowski and Krolikowski (1986). The original equations were only valid for saturated liquid feed $(q=1)$, but this has been extended to include any liquid fraction $(q)$ and the result is very simple. For sharp product splits and normalized feed, the minimum reflux value for the Petlyuk column is given by:

$$
L_{\min }=\max \left(\frac{\alpha_{A} z_{A}}{\alpha_{A}-\phi_{1}}, \frac{\alpha_{A} \phi_{2}}{\alpha_{A}-\phi_{2}}+\frac{\alpha_{B} z_{B}}{\alpha_{B}-\phi_{2}}\right)
$$

The roots $\left(\phi_{1}, \phi_{2}\right)$ are solutions of the Underwood equation for the prefractionator feed:

$$
\frac{\alpha_{A} z_{A}}{\alpha_{A}-\phi}+\frac{\alpha_{B} z_{B}}{\alpha_{B}-\phi}+\frac{\alpha_{C} z_{C}}{\alpha_{C}-\phi}=(1-q)
$$

Note that the Underwood roots obeys the following inequality: $\alpha_{A}>\phi_{1}>\alpha_{B}>$ $\phi_{2}>\alpha_{c}$.

The prefractionator has a V-shaped minimum reflux characteristic $L_{1},=L_{1, \min }(\beta)$ as shown in the lower part of Figure A.1 and for sharp a/c split it can be expressed

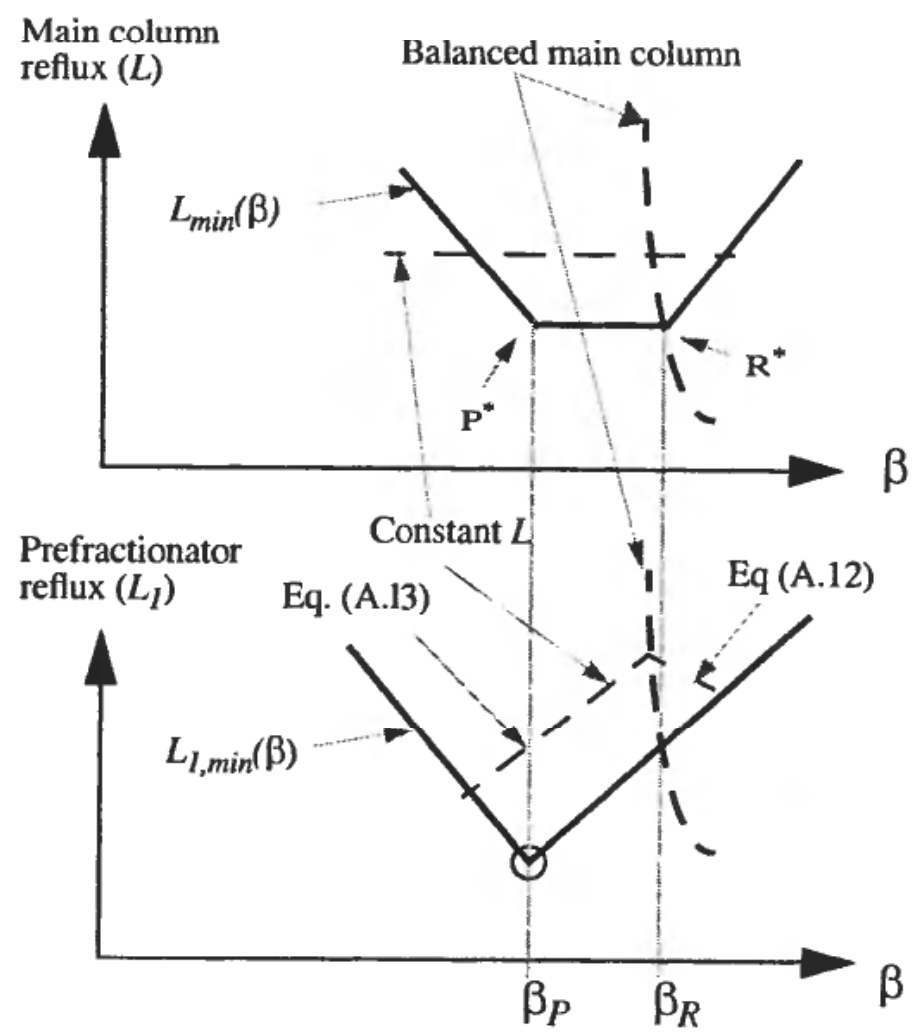

Figure A.1. Minimum reflex for the whole Petlyuk column $(L)$ has a flat minimum region $\left(\mathrm{P}^{*} \mathrm{R}^{*}\right)$ for recoveries in the range between the preferred split $\left(\beta_{P}\right)$ and a balanced main column $\left(\beta_{R}\right)$, while minimum reflux $\left(L_{1}\right)$ for the prefactionator itself has a sharp minimum at the preferred split. 
analytically by:

$$
L_{1, \min }(\beta)=\left\{\begin{array}{lll}
\frac{\alpha_{A} \phi_{1}}{\alpha_{A}-\phi_{1}}+\frac{\alpha_{B} \phi_{1} \beta}{\alpha_{B}-\phi_{1}} & \text { for } & \beta \leqslant \beta_{P} \\
\frac{\alpha_{A} \phi_{2}}{\alpha_{A}-\phi_{2}}+\frac{\alpha_{B} \phi_{2} \beta}{\alpha_{B}-\phi_{2}} & \text { for } & \beta \geqslant \beta_{P}
\end{array}\right.
$$

Equation (A.9) has a distinct minimum which represent the absolute minimum energy operating point for the prefractionator: this is denoted the preferred split (Stichlmair, 1988). Analytical values for prefractionator reflux $\left(L_{1, p}\right)$ and middle key recovery $\left(\beta_{P}\right)$ at the preferred split, can be found by equating the two straight lines of equation (A.9). Note that in general, $\beta_{P}$ is dependent of feed composition and liquid fraction via equation (A.8), but in the special case of saturated liquid, $\beta_{P}$ is only dependent on the relative volatilities:

$$
\beta_{P}=\frac{\alpha_{B}-\alpha_{C}}{\alpha_{A}-a_{C}} \quad \text { for } \quad q=1
$$

Further elaboration of the result show that the minimum energy for the whole Petlyuk column occurs not at a single point, but is constant in the range of fractional recoveries $(\beta)$ between the preferred split $\left(\beta_{P}\right)$, which yields minimum energy consumption in the prefractionator, and for a certain $\beta=\beta_{R}$, for which we will find that the minimum energy requirement is fulfilled at the same time for both the upper and lower parts of the main column, also denoted: a balanced main column. The prefractionator has to be operated at its minimum characteristics: $L_{1,}=L_{1, \min }(\beta)$ equation (A.9), with $\beta$ between $\beta_{P}$ and $\beta_{R}$. We may have three different cases: 1) $\beta_{P}>\beta_{R}$, 2) $\beta_{P}<\beta_{R}$ and 3) $\beta_{P}=\beta_{R}$, where the last one is a special case where the solution is reduced to a single point in the $\left(\beta, L_{1}\right)$-plane at the preferred split. Figure A.1 shows an example where $\beta_{P}<\beta_{R}$.

The analytical expression in equation (A.7) is deduced by requiring minimum reflux in the prefractionator and in the main column. The main column can be regarded as two binary columns separating components $a / b$ and $b / c$. Since the columns are connected, we cannot specify the reflux in each part freely, thus when we set the main column reflux $(L)$ and the two degrees of freedom (here $\beta$ and $L_{1}$ ) all other flows are determined. Minimum reflux requirement can then be expressed in these three variables for both parts of the main column.

We can find a function $L_{\min }^{\text {upper }}\left(\beta, L_{1}\right)$ which gives the minimum reflux requirement (into the main column top) when we only consider the upper part of the main column, and similarly $L_{\min }^{\text {lower }}\left(\beta, L_{1}\right)$ gives the minimum reflux requirement (into the main column top) when we only consider the lower part of the main column. Then the main column minimum reflux as given in equation (A.7) can be found by solving

$$
\begin{gathered}
L_{\min }\left(\beta, L_{1}\right)=\max \left(_{\min }^{\text {upper }}\left(\beta, L_{1}\right), L_{\min }^{\text {lower }}\left(\beta, L_{1}\right)\right) \\
\text { subject to } L_{1} \geqslant L_{1, \min }(\beta)
\end{gathered}
$$

The properties of the solution surface $L_{\min }\left(\beta, L_{1}\right)$ can be studied further by considering each of $L_{\min }^{\text {upper }}\left(\beta, L_{1}\right)$ and $L_{\min }^{\text {lower }}\left(\beta, L_{1}\right)$. Fortunately, these functions are found to be linear in $\beta$ and $L_{1}$. Thus we can express these functions as straight lines in the $\left(\beta, L_{1}\right)$ plane for a constant $L$. Solved with respect to the prefractionator reflux $\left(L_{1}\right)$ we can find the simple analytic expressions in equation (A.12) with $L_{\min }^{\text {upper }}$ and equation (A.13) with $L_{\min }^{\text {lower }}$. 


$$
\begin{gathered}
L_{1}=L_{\min }^{\text {upper }}-\frac{\beta z_{B} \alpha_{A}}{\left(\alpha_{A}-\alpha_{B}\right)-\frac{z_{A} a_{B}}{L_{\min }^{\text {upper }}}} \\
L_{1}=L_{\min }^{\text {lower }}-z_{B}-\frac{(1-\beta) z_{B} \alpha_{C}}{\left(\alpha_{B}-\alpha_{C}\right)-\frac{z_{C} a_{B}}{L_{\min }^{\text {lower }}+z_{A}+z_{C}-(1-q)}}
\end{gathered}
$$

Note that these equations are only valid when there is a pinch zone around the corresponding main column "feed" location and we have sharp $a / c$ and $b / c$ splits in the two main column parts.

We can interpret equation (A.12) as a level contour for the surface $L=L_{\min }^{\text {upper }}\left(\beta, L_{1}\right)$ in the $\left(\beta, L_{1}\right)$-plane when we only consider the minimum reflux requirement for the upper part of the main column. Similarly equation (A.13) represents a contour line for $L=L_{\min }^{\text {lower }}\left(\beta, L_{1}\right)$ in the $\left(\beta, L_{1}\right)$-plane when we only consider the minimum reflux requirement for the lower part.

The operating points in the $\left(\beta, L_{1}\right)$-plane for a balanced main column $\left(L_{1, R}, \beta_{R}\right)$ are found at the intersection of the lines described by equations (A.12) and (A.13) for the same main column reflux $\left(L=L_{\min }^{\text {lower }}=L_{\min }^{\text {upper }}\right)$. For the case of saturated liquid feed $(q=1)$, the solution can be expressed by

$$
\begin{aligned}
\beta_{R} & =\frac{L\left(\alpha_{A}-\alpha_{B}\right)-F z_{A} \alpha_{B}}{L \alpha_{c}-\left(L+F\left(z_{A}+z_{C}\right)\right)} \\
L_{1, R} & =L\left(1-\frac{z_{B} \alpha_{A}}{L \alpha_{A}-\left(L+z_{A}+z_{C}\right) \alpha_{C}}\right)
\end{aligned}
$$

The reason for the flat optimum (see (Figure A.1) is that the level lines given by equations (A.12) and (A.13) coincide with the corresponding branches of the minimum reflux characteristic for the prefractionator equation (A.9) at the optimum. The proof for $q \neq 1$ follow the same procedure as in Fidkowski and Krolikowski (1986). The result is the simple analytical expression for the overall minimum reflux in equation (A.7) which is valid also for any liquid fraction $(q)$.

We might have expected the optimum to be at the preferred prefractionator split $\left(\mathrm{P}^{*}\right)$ or at a balanced main column $\left(\mathrm{R}^{*}\right)$. The fact that all points on the straight line $\mathrm{P}^{*} \mathrm{R}^{*}$ are optimal is very important.

\section{A.3. Mapping $V\left(\beta, L_{1}\right)$ to $V\left(R_{l}, R_{v}\right)$}

We here consider the surface $V\left(R_{l}, R_{v}\right)$ for the case with infinite number of stages. From equations (A.12) and (A.13) we see that for a fixed reflux $(L)$, the level contour of $L\left(\beta, L_{1}\right)$ (and then also $\left.V\left(\beta, L_{1}\right)\right)$ are straight line segments in the $\left(\beta, L_{1}\right)$-plane (see the dashed level line for constant $L$ in Figure A.1 which represent operating lines from equations (A.12) and (A.13)). Recall also the definition of the split ratios, and observe how $R_{v}$ can be expressed as a function of $L, L_{1}$, and $\beta$ in the case of sharp product splits (feed is normalized):

$$
R_{l}=\frac{L_{1}}{L} \quad R_{v}=\frac{V_{2}}{V}=\frac{L_{1}+z_{a}+z_{b} \beta-(1-q)}{L+z_{a}+(1-q)}
$$


Thus, for constant reflux $(L)$, any straight line in the $\left(\beta, L_{1}\right)$-plane map to a straight line in the $\left(R_{l}, R_{v}\right)$-plane.

The optimum which occur on a line segment in the $\left(\beta, L_{1}\right)$-plane will then also be a straight line segment in the $\left(R_{l}, R_{v}\right)$-plane. Fidkowski's equations, extended to handle any feed liquid fraction $(q)$, together with equation (A.15) gives us the tool to compute all possible level lines on the surface $V\left(R_{l}, R_{v}\right)$ with the feed composition, liquid fraction and component relative volatilities as parameters.

Each level line is a polygon with four characteristic corners:

Cl. Operating the prefractionator at preferred split and minimum reflux $\left(L_{1, P}, \beta_{P}\right)$, over-refluxing the main column $\left(L>L_{\min }\right)$.

C2. Operating along the left branch of the prefractionator characteristic $\left(L_{1}=L_{1, \min }(\beta), \beta<\beta_{P}\right), L$ from intersection of equations (A.9) and (A.13)

C3. Operating where the main column is balanced $\left(L_{1, R}, \beta_{R}\right)$, while the prefractionator is over-refluxed $\left(L_{1}>L_{1, \min }(\beta)\right)$

C4. Operating along the right branch the prefractionator, above the balance point $\left(L_{1}=L_{1, \min }(\beta), \beta>\beta_{R}\right), L$ from intersection of equations (A.9) and (A.12)

Note that corner lines $\mathrm{C} 1, \mathrm{C} 2$ and $\mathrm{C} 3, \mathrm{C} 4$ coincide at each end of the optimum line in the $\left(R_{l}, R_{v}\right)$-plane. (The list items above are valid for $\beta_{P}<\beta_{R}$. In the case of $\beta_{P}>\beta_{R}$ we have to reformulate item 2 and 4.)

It is interesting to observe that the point $\left(\beta_{P}, L_{1, P}\right)$ map to a curve in the $\left(R_{l}, R_{v}\right)$ plane when we increase the main column reflux (Corner 1). And for $q=1$, operating along the right branch of the prefractionator, above the balance point (Corner 4) map into a single point in the $\left(R_{l}, R_{v}\right)$-plane.

The constant energy level lines from corner 2 via corner 3 to corner 4 are directly described by the equations (A.12) and (A.13).

\section{A.4. Nomenclature}

$B$ bottom product flow

$D$ top product flow

d disturbance

$F \quad$ feed flow

$L \quad$ liquid flow

$R_{l} \quad$ liquid split fraction

$R_{v} \quad$ vapor split fraction

$S \quad$ side-stream flow

$T \quad$ temperature

$V \quad$ vapor flow

$q \quad$ feed liquid fraction

$x \quad$ liquid mole fraction

$y \quad$ vapor mole fraction, measurement

$z \quad$ feed composition

$u \quad$ control input vector

$r$ recovery

$f, g, h$ functions

$t \quad$ time

$w \quad$ material flow

$\alpha \quad$ relative volatility

$\beta \quad b$-component recovery in prefractionator top 


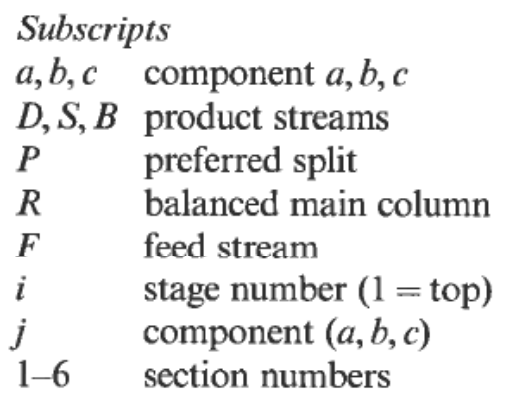

\section{REFERENCES}

Christiansen, A. C. and Skogestad, S. (1997). Energy savings in integrated Petlyuk distillation arrangements. Importance of using the preferred separation, AIChE Annual meeting, Los Angeles, November 1997. Paper 199d, updated version as found in CHRISTIANSEN (1997).

ChristianSEN, A. C. (1997). "Studies on optimal design and operation of integrated distillation arrangements", PhD thesis 1997: 149, Norwegian University of Science and Technology (NTNU).

FIDKOWSKI, Z. and KROLIKOwSKI, L. (1986). Thermally coupled system of distillation columns: optimization procedure, AIChE Journal, Vol. 32, No. 4.

Halvorsen, I. J. (2001). Minimum Energy Requirements in Complex Distillation Arrangements. NTNU Dr. ing. Thesis, 2001:43. Available from http://kikp.chembio.ntnu.no/ thesis/download/2001/halvorsen/

Skogestad, S. and Postlethwaite, I. (1996). Multivariable Feedback Control, Wiley.

Stichlmair, J. (1988). Distillation and rectification, Ullmann's Encyclopedia of Industrial Chemistry, B3, 4-1-4-94.

Triantafyllou, C. and SMITH, R. (1992). The design and operation of fully thermally coupled distillation columns, Trans. IChemE 7-(part A), 118-132.

Wolff, E., Skogestad, S. and Havre, K. (1994). Dynamics and Control of Integrated Threeproduct (Petlyuk) Distillation Columns, ESCAPE'4, Dublin.

WolFF, E. and SKOGESTAD, S. (1996). Operation of integrated three-product (Petlyuk) distillation columns, Ind. Eng. Chem. Res., 34, $2094-2103$. 